A. Jourani (1996), Subdifferentiability and subdifferential monotonicity of $\gamma$-paraconve functions, Control Cybernet. 25, 721-737.

D. Pallaschke and S. Rolewicz (1997), Foundations of Mathematical Optimization, Math. Appl. 388, Kluwer, Dordrecht.

R. T. Rockafellar (1970), On the maximal monotonicity of subdifferential mappings, Pacific J. Math. 33, 209-216.

R. T. Rock afellar (1980), Generalized directional derivatives and subgradients of non convex functions, Canad. J. Math. 32, 257-280.

S. Rolewicz (1999), On $\alpha(\cdot)$-monotone multifunctions and differentiability of $\gamma$-paraconvex functions, Studia Math. 133, 29-37.

Institute of Mathematics

Polish Academy of Sciences

Śniadeckich 8, P.O. Box 137

00-950 Warszawa, Poland

E-mail: rolewicz@impan.gov.pl

\section{On the complemented subspaces of the Schreier spaces}

by

I. GASPARIS (Stillwater, OK) and D. H. LEUNG (Singapore)

Abstract. It is shown that for every $1 \leq \xi<\omega$, two subspaces of the Schreier space $X^{\xi}$ generated by subsequences $\left(e_{l_{n}}^{\xi}\right)$ and $\left(e_{m_{n}}^{\xi}\right)$, respectively, of the natural Schauder basis $\left(e_{n}^{\xi}\right)$ of $X^{\xi}$ are isomorphic if and only if $\left(e_{l_{n}}^{\xi}\right)$ and $\left(e_{m_{n}}^{\xi}\right)$ are equivalent. Further, $X^{\xi}$ admits a continuum of mutually incomparable complemented subspaces spanned by subsequences of $\left(e_{n}^{\xi}\right)$. It is also shown that there exists a complemented subspace spanned by a block basis of $\left(e_{n}^{\xi}\right)$, which is not isomorphic to a subspace generated by a subsequence of $\left(e_{n}^{\zeta}\right)$, for every $0 \leq \zeta \leq \xi$. Finally, an example is given of an uncomplemented subspace of $X^{\xi}$ which is spanned by a block basis of $\left(e_{n}^{\xi}\right)$

1. Introduction. The Schreier families $\left\{S_{\xi}\right\}_{\xi<\omega_{1}}$ of finite subsets of positive integers (the precise definition is given in the next section), introduced in [1], have played a central role in the development of modern Banach space theory. We mention the use of Schreier families in the construction of mixed Tsirelson spaces which are asymptotic $\ell_{1}$ and arbitrarily distortable [3]. The distortion of mixed Tsirelson spaces has been extensively studied in [2]. In that paper, as well as in [14], the moduli $\left(\delta_{\alpha}\right)_{\alpha<\omega_{1}}$ were introduced measuring the complexity of the asymptotic $\ell_{1}$ structure of a Banach space. The definitions of those moduli also involve the Schreier families. Other applications can be found in [6] and [5] where the Schreier families form the main tool for detcrmining the structure of those convex combinations of a weakly null sequence that tend to zero in norm, or are equivalent to the unit vector basis of $c_{0}$. For applications of the Schreier families in the construction of hereditarily indecomposable Banach spaces, we refer to [3] and [4].

A notion companion to the Schreier families is that of the Schreier spaces. These are Banach spaces whose norm is related to a corresponding Schreier family. More precisely, for every countable ordinal $\xi$, we define a norm $\|\cdot\|_{\xi}$ on $c_{00}$, the space of finitely supported real-valued sequences, in the following

2000 Mathematics Subject Classification: Primary 46B03; Secondary 46B15, $03 \mathrm{E} 10$. Key words and phrases: complemented subspace, Schreier sets. 
manner: Given $x=(x(n)) \in c_{00}$ define

$$
\|x\|_{\xi}=\sup _{F \in S_{\xi}} \sum_{n \in F}|x(n)| .
$$

$X^{\xi}$, the Schreier space of order $\xi$, is the completion of $c_{00}$ under the norm $\|\cdot\|_{\xi} \cdot X^{0}=c_{0}$, the Banach space of null sequences. $X^{1}$ was first considered by Schreier [15] in order to provide an example of a weakly null sequence without Cesàro summable subsequence. It is proven in [1] that the natural Schauder basis $\left(e_{n}^{\xi}\right)$ of $X^{\xi}$ is 1-unconditional and shrinking. $X^{1}$ has been studied in [13] where it is shown that every quotient of $X^{1}$ is $c_{0}$-saturated. That is, every infinite-dimensional subspace contains a further subspace isomorphic to $c_{0}$.

Given $M$, an infinite subset of $\mathbb{N}$, we let $X_{M}^{\xi}$ denote the closed linear subspace of $X^{\xi}$ spanned by the subsequence $\left(e_{n}^{\xi}\right)_{n \in M}$. For an element $x \in$ $X^{\xi}, x=\sum_{n \in \mathbb{N}} a_{n} e_{n}^{\xi}$, we set $\|x\|_{0}=\sup _{n \in \mathbb{N}}\left|a_{n}\right|$. The main result of this paper is the following

THEOREM 1.1. Let $L=\left(l_{n}\right), M=\left(m_{n}\right)$ be infinite subsets of $\mathbb{N}$, and let $\xi<\omega$. The following are equivalent:

1. There exist a bounded linear operator $T: X_{L}^{\xi} \rightarrow X_{M}^{\xi}$ and $\delta>0$ such that $\left\|T\left(e_{l}^{\xi}\right)\right\|_{0}>\delta$ for all $l \in L$.

2. $\left(e_{l_{n}}^{\zeta}\right)$ dominates $\left(e_{m_{n}}^{\zeta}\right)$ for every $\zeta \leq \xi$.

3. $\left(e_{l_{n}}^{\xi}\right)$ dominates $\left(e_{m_{n}}^{\xi}\right)$.

We recall here that a basic sequence $\left(x_{n}\right)$ in some Banach space $X$ is said to dominate the basic sequence $\left(y_{n}\right)$ in the Banach space $Y$ if there exists a constant $C>0$ so that $\left\|\sum_{i=1}^{n} a_{i} y_{i}\right\| \leq C\left\|\sum_{i=1}^{n} a_{i} x_{i}\right\|$ for every $n \in \mathbb{N}$ and all scalar sequences $\left(a_{i}\right)_{i=1}^{n}$. Equivalently, $\left(x_{n}\right)$ dominates $\left(y_{n}\right)$ if there exists a bounded linear operator $T$ from the closed linear span of $\left(x_{n}\right)$ into the closed linear span of $\left(y_{n}\right)$ so that $T\left(x_{n}\right)=y_{n}$ for every $n \in \mathbb{N}$. The sequences $\left(x_{n}\right)$ and $\left(y_{n}\right)$ are equivalent if each one of them dominates the other.

Combining Theorem 1.1 with Lemma 3.14, we obtain of $\mathbb{N}$.

CoRollaRY 1.2. Let $\xi<\omega$ and $L=\left(l_{n}\right), M=\left(m_{n}\right)$ be infinite subsets

1. If $X_{L}^{\xi}$ is isomorphic to a subspace of $X_{M}^{\xi}$ then $\left(e_{l_{n}}^{\xi}\right)$ dominates $\left(e_{m_{n}}^{\xi}\right)$. Consequently, $X_{L}^{\xi}$ is isomorphic to $X_{M}^{\xi}$ if, and only if, $\left(e_{l_{n}}^{\xi}\right)$ is equivalent to $\left(e_{m_{n}}^{\xi}\right)$. $\zeta \leq \xi$
3. Suppose that $\left(e_{l_{n}}^{\xi}\right)$ dominates a permutation of $\left(e_{m_{n}}^{\xi}\right)$. Then $\left(e_{l_{n}}^{\xi}\right)$ dominates $\left(e_{m_{n}}^{\xi}\right)$.

Theorem 1.1 combined with elementary descriptive set theory yields our next result on the structure of the subsequences of $\left(e_{n}^{\xi}\right), \xi<\omega$. We recall here that the Banach spaces $X$ and $Y$ are incomparable if neither of them is isomorphic to a closed linear subspace of the other. In what follows, $[\mathbb{N}]$ denotes the set of all infinite subsets of $\mathbb{N}$.

TheOREM 1.3. For every $1 \leq \xi<\omega$ there exists $\mathcal{A} \subset[\mathbb{N}]$ (depending on $\xi$ ) of cardinality equal to the continuum and with the following property: for every pair $(L, M)$ of distinct elements of $\mathcal{A}$, the spaces $X_{L}^{\xi}$ and $X_{M}^{\xi}$ are incomparable.

Corollary 3.15 provides another application of Theorem 1.1: $X_{N}^{\xi}$ is not primary, for every $N \in[\mathbb{N}]$ and all $1 \leq \xi<\omega$.

The proofs of the aforementioned results are given in the third section of our paper. In the fourth section we deal with complemented subspaces of $X^{\xi}$ spanned by block bases of $\left(e_{n}^{\xi}\right)$. Proposition 4.3 and Corollary 4.4 establish that for every semi-normalized weakly null sequence in $X^{\xi}, \xi<\omega$, there exist $\zeta \leq \xi$ and a subsequence which is equivalent to a subsequence of $\left(e_{n}^{\zeta}\right)$

Further, we show that there exists a block basis of $\left(e_{n}^{\xi}\right)$ spanning a complemented subspace of $X^{\xi}$ which is not isomorphic to $X_{M}^{\zeta}$, for all $0 \leq \zeta \leq \xi$ and every infinite subset $M$ of $\mathbb{N}$. We also show that there exists a block basis of $\left(e_{n}^{\xi}\right)$ spanning a subspace which is not complemented in $X^{\xi}$.

The problem of the isomorphic classification of the complemented subspaces of $X^{\xi}$, even for block subspaces, seems rather difficult.

Part of the research for this paper was conducted while the second author visited the University of Texas at Austin. The second author thanks the Department of Mathematics there, especially the Banach space group, for making the visit possible. Thanks are also due to Ted Odell for several conversations rogarcling the results contained herein.

2. Preliminaries. We shall make use of standard Banach space facts and terminology as may be found in [11]. In this section we shall review some of the necessary concepts. We shall also review two important hierarchies, the Schreicr bierarchy [1] and the repeated averages hierarchy [6]. Finally we shall static some fundamental results from descriptive set theory which will be widely used in what follows. For a detailed study of descriptive set theory we refer to [9].

We first indicate some special notation that we will be using. A sequence $\left(x_{n}\right)_{n=1}^{\infty}$ of elements of an arbitrary set will be conveniently denoted by 
$\left(x_{n}\right)$. Given $M$, a subset of $\mathbb{N},[M]^{<\infty}$ denotes the set of all finite subsets of $M$, while $[M]$ stands for the set of all infinite subsets of $M$. If $M \in[\mathbb{N}$, then the notation $M=\left(m_{n}\right)$ indicates that $M=\left\{m_{1}<m_{2}<\ldots\right\}$. Let $E, F$ be finite sets of integers. We shall adopt the notation $E<F$ to mean $\max E<\min F$. If $x=(x(n))$ belongs to $c_{00}$, the space of finitely supported real-valued sequences, and $F \in[\mathbb{N}]^{<\infty}$, then $x(F)=\sum_{n \in F} x(n)$, and $|x|(F)=\sum_{n \in F}|x(n)|$.

All Banach spaces considered throughout this paper are real. $\ell_{1}$ denotes the Banach space of absolutely summable sequences under the norm given by the sum of the absolute values of the coordinates. $c_{0}$ is the Banach space of null sequences under the norm given by the maximum of the absolute values of the coordinates. By the term subspace of a Banach space we shall always mean a closed linear subspace. A subspace $Y$ of the Banach space $X$ is said to be complemented if it is the range of a bounded linear projection on $X$.

We next recall that if $\left(x_{n}\right)$ is a sequence in some normed linear space, then the sequence $\left(y_{n}\right)$ is called a block subsequence (resp. convex block subsequence) of $\left(x_{n}\right)$ if there exist sets $F_{i} \subset \mathbb{N}$ with $F_{1}<F_{2}<\ldots$ and a sequence $\left(a_{i}\right)$ of scalars (resp. non-negative scalars such that $\sum_{n \in F_{i}} a_{n}=1$, for every $i \in \mathbb{N}$ ) such that for every $i \in \mathbb{N}, y_{i}=\sum_{n \in F_{i}} a_{n} x_{n}$. We then denote by supp $y_{i}$ the support of $y_{i}$, that is, the set $\left\{n \in F_{i}:\left|a_{n}\right|>0\right\}$. We shall also adopt the notation $y_{1}<y_{2}<\ldots$ to indicate that $\left(y_{n}\right)$ is a block subsequence of $\left(x_{n}\right)$. If $\left(x_{n}\right)$ is Schauder basic, then $\left(y_{n}\right)$ will be called a block basis (resp. convex block basis) of $\left(x_{n}\right)$.

Next we review the definition and some basic properties of the Schreier families $\left\{S_{\xi}\right\}_{\xi<\omega_{1}}$ (cf. [1]). The Schreier families are defined by transfinite induction as follows: $S_{0}=\{\{n\}: n \in \mathbb{N}\} \cup\{\emptyset\}$. Suppose $S_{\zeta}$ has been defined for every $\zeta<\xi$. If $\xi$ is a successor ordinal, say $\xi=\zeta+1$, we set

$$
\begin{aligned}
S_{\xi}=\left\{\bigcup_{i=1}^{n} F_{i}: n \in \mathbb{N},\right. & n \leq \min F_{1}, \\
& \left.F_{1}<\ldots<F_{n}, F_{i} \in S_{\zeta}, i \leq n\right\} \cup\{\emptyset\} .
\end{aligned}
$$

If $\xi$ is a limit ordinal, let $\left(\xi_{n}\right)$ be a preassigned increasing sequence of successor ordinals whose limit is $\xi$. We set

$$
S_{\xi}=\bigcup_{n=1}^{\infty}\left\{F \in S_{\xi_{n}}: n \leq \min F\right\} \cup\{\emptyset\} .
$$

Given $M \in[\mathbb{N}]$ we denote by $S_{\xi}[M]$ the family $\left\{F: F \in S_{\xi}, F \subset M\right\}$.

An important property shared by the Schreier families is that they are spreading: If $\left\{p_{1}, \ldots, p_{k}\right\} \in S_{\xi}, p_{1}<\ldots<p_{k}$, and $q_{1}<\ldots<q_{k}$ are so that $p_{i} \leq q_{i}$ for all $i \leq k$, then $\left\{q_{1}, \ldots, q_{k}\right\} \in S_{\xi}$.
Of particular interest are the maximal (under inclusion) members of $S_{\xi}$. The following lemma concerning those sets is proved in [8].

LEMMA 2.1. Let $M \in[\mathbb{N}]$ and $\xi<\omega_{1}$. Then there exists a (necessarily) unique sequence $\left\{F_{n}^{\xi}(M)\right\}_{n=1}^{\infty}$ of successive maximal $S_{\xi}$ sets so that $M=$ $\bigcup_{n=1}^{\infty} F_{n}^{\xi}(M)$.

REMARK. The following stability properties of $\left\{F_{n}^{\xi}(M)\right\}_{n=1}^{\infty}$ are easily verified:

1. If $k_{1}<k_{2}<\ldots$ and $N=\bigcup_{n=1}^{\infty} F_{k_{n}}^{\xi}(M)$, then $F_{n}^{\xi}(N)=F_{k_{n}}^{\xi}(M)$ for all $n \in \mathbb{N}$.

2. Let $M=\left(m_{i}\right)$ and $N=\left(n_{i}\right)$ be infinite subsets of $\mathbb{N}$. Assume that for some $p \in \mathbb{N}, m_{i}=n_{i}$ for all $i \leq p$. If $F_{k}^{\xi}(M)$ is contained in $\left\{m_{i}: i \leq p\right\}$, then $F_{i}^{\xi}(M)=F_{i}^{\xi}(N)$ for all $i \leq k$.

We shall make use of the following

Lemma 2.2. Let $M \in[\mathbb{N}], L \in[M]$ and $\xi<\omega$. Then $\max F_{1}^{\xi}(M) \leq$ $\max F_{1}^{\xi}(L)$.

Proof. Suppose $L=\left(l_{i}\right)$ and $M=\left(m_{i}\right)$. We prove the assertion by induction on $\xi$. The case $\xi=0$ is trivial. Assume now that $\xi \geq 1$ and that the assertion holds for $\xi-1$ and all $P, Q$ with $Q \in[P]$.

For an arbitrary $P \in[\mathbb{N}]$, we set $P_{1}=P$ and

$$
P_{i}=\left\{p \in P: p>\max F_{i-1}^{\xi-1}(P)\right\}, \quad i \geq 2 .
$$

We observe that $F_{i}^{\xi-1}(P)=F_{1}^{\xi-1}\left(P_{i}\right)$ for all $i \in \mathbb{N}$. We also have $F_{1}^{\xi}(P)=$ $\bigcup_{i=1}^{p_{1}} F_{1}^{\xi-1}\left(P_{i}\right)$, where $p_{1}=\min P_{1}$. It follows now, by the induction hypothesis, that $L_{i} \in\left[M_{i}\right]$ for all $i \in \mathbb{N}$. Therefore,

$$
\max F_{1}^{\xi}(M)=\max F_{1}^{\xi-1}\left(M_{m_{1}}\right) \leq \max F_{1}^{\xi-1}\left(L_{m_{1}}\right) \leq \max F_{1}^{\xi}(L)
$$

\section{as $m_{1} \leq l_{1}$.}

We now pass to the definition of the repeated averages hierarchy introduced in [6]. We let $\left(e_{n}\right)$ denote the unit vector basis of $c_{00}$. For every countable ordinal $\xi$ and every $M \in[\mathbb{N}]$, we define a convex block subsequence $\left(\xi_{n}^{M}\right)_{n=1}^{\infty}$ of $\left(e_{n}\right)$ by transfinite induction on $\xi$ in the following manner: If $\xi=0$, then $\xi_{n}^{M}=e_{m_{n}}$, for all $n \in \mathbb{N}$, where $M=\left(m_{n}\right)$. Assume that $\left(\zeta_{n}^{M}\right)_{n=1}^{\infty}$ has been defined for all $\zeta<\xi$ and $M \in[\mathbb{N}]$. Let $\xi=\zeta+1$. Set

$$
\xi_{1}^{M}=\frac{1}{m_{1}} \sum_{i=1}^{m_{1}} \zeta_{i}^{M}
$$

where $m_{1}=\min M$. Suppose that $\xi_{1}^{M}<\ldots<\xi_{n}^{M}$ have been defined. Let

$$
M_{n}=\left\{m \in M: m>\max \operatorname{supp} \xi_{n}^{M}\right\} \quad \text { and } \quad k_{n}=\min M_{n} .
$$


Set

$$
\xi_{n+1}^{M}=\frac{1}{k_{n}} \sum_{i=1}^{k_{n}} \zeta_{i}^{M_{n}}
$$

If $\xi$ is a limit ordinal, let $\left(\xi_{n}+1\right)$ be the sequence of ordinals associated with $\xi$ in the definition of $S_{\xi}$, and let $M \in[\mathbb{N}]$. Define

$$
\xi_{1}^{M}=\left[\xi_{m_{1}}+1\right]_{1}^{M}
$$

where $m_{1}=\min M$. Suppose that $\xi_{1}^{M}<\ldots<\xi_{n}^{M}$ have been defined. Let

$$
M_{n}=\left\{m \in M: m>\max \operatorname{supp} \xi_{n}^{M}\right\} \quad \text { and } \quad k_{n}=\min M_{n} .
$$

Set

$$
\xi_{n+1}^{M}=\left[\xi_{k_{n}}+1\right]_{1}^{M_{n}} .
$$

The inductive definition of $\left(\xi_{n}^{M}\right)_{n=1}^{\infty}, M \in[\mathbb{N}]$, is now complete.

The following properties are established in [6].

P1 $\quad\left(\xi_{n}^{M}\right)_{n=1}^{\infty}$ is a convex block subsequence of $\left(e_{n}\right)$ such that, for all $M$ $\epsilon[\mathbb{N}]$ and $\xi<\omega_{1}$, we have $M=\bigcup_{n=1}^{\infty} \operatorname{supp} \xi_{n}^{M}$.

P2 $\operatorname{supp} \xi_{n}^{M} \in S_{\xi}$ for all $M \in[\mathbb{N}], \xi<\omega_{1}$ and $n \in \mathbb{N}$.

P3 If $M, N \in[\mathbb{N}], \xi<\omega_{1}$, and $\operatorname{supp} \xi_{i}^{M}=\operatorname{supp} \xi_{i}^{N}$ for $i \leq k$, then $\xi_{i}^{M}=\xi_{i}^{N}$ for $i \leq k$

P4 If $\xi<\omega_{1},\left\{n_{k}: k \in \mathbb{N}\right\} \subset \mathbb{N}$, and $\left\{L_{k}: k \in \mathbb{N}\right\} \subset[\mathbb{N}]$ are such that $\operatorname{supp} \xi_{n_{i}}^{L_{i}}<\operatorname{supp} \xi_{n_{i+1}}^{L_{i+1}}$ for all $i \in \mathbb{N}$, then letting $L=\bigcup_{i=1}^{\infty} \operatorname{supp} \xi_{n_{i}}^{L_{i}}$, we have $\xi_{i}^{L}=\xi_{n_{i}}^{L_{i}}$, for all $i \in \mathbb{N}$

Properties P3 and P4 are called stability properties of the hierarchy $\left\{\left(\xi_{n}^{M}\right)_{n=1}^{\infty}: M \in[\mathbb{N}]\right\}$. It is easily seen, by induction, that $\operatorname{supp} \xi_{n}^{M}=F_{n}^{\xi}(M)$ for every $\xi<\omega$, all $M \in[\mathbb{N}]$ and $n \in \mathbb{N}$.

In the next lemma we show that for $\xi<\omega$ and $M \in[\mathbb{N}]$ the sequence $\left(\xi_{n}^{M}\right)$, considered as a sequence in $X^{\xi}$, is equivalent to the unit vector basis of $c_{0}$. Moreover, the equivalence constant depends only on $\xi$.

LEMMA 2.3. $\left\|\sum_{i=1}^{n} \xi_{i}^{M}\right\|_{\xi} \leq \xi+1$ for every $M \in[\mathbb{N}], n \in \mathbb{N}$, and $\xi<\omega$.

Proof. We use induction on $\xi$. The case $\xi=0$ is trivial. Assume the assertion holds for $\xi-1$. Let $G \in S_{\xi}$. We shall show that $\sum_{i=1}^{n} \xi_{i}^{M}(G) \leq \xi+1$, for every $M \in[\mathbb{N}]$ and $n \in \mathbb{N}$. To this end choose successive members $G_{1}<\ldots<G_{l}$, of $S_{\xi-1}$ so that $l \leq \min G$ and $G=\bigcup_{i=1}^{l} G_{i}$. Let $\left\{i_{1}, \ldots, i_{p}\right\}$ be an enumeration of the set $\left\{i \leq n: F_{i}^{\xi}(M) \cap G \neq \emptyset\right\}$. We define

$$
L=\bigcup_{t=1}^{p} F_{i_{t}}^{\xi}(M) \cup\left\{m \in M: m>\max F_{i_{p}}^{\xi}(M)\right\}
$$

and observe that $F_{i_{t}}^{\xi}(M)=\bigcup_{j=r_{t-1}+1}^{r_{t}} F_{j}^{\xi-1}(L)$ for all $t \leq p$, where $r_{0}=$ $0<r_{1}<\ldots<r_{p}$ are chosen so that $r_{t}-r_{t-1}=\min F_{i_{t}}^{\xi}(M)$ for all $t \leq p$.
Therefore,

$$
\xi_{i_{t}}^{M}=\frac{1}{\min F_{i_{t}}^{\xi}(M)} \sum_{j=r_{t-1}+1}^{r_{t}}(\xi-1)_{j}^{L}, \quad t \leq p
$$

and thus

$$
\begin{aligned}
\sum_{t=2}^{p} \xi_{i_{t}}^{M}(G) & =\sum_{t=2}^{p} \sum_{s=1}^{l} \frac{1}{\min F_{i_{t}}^{\xi}(M)} \sum_{j=r_{t-1}+1}^{r_{t}}(\xi-1)_{j}^{L}\left(G_{s}\right) \\
& =\sum_{s=1}^{l} \sum_{t=2}^{p} \frac{1}{\min F_{i_{t}}^{\xi}(M)} \sum_{j=r_{t-1}+1}^{r_{t}}(\xi-1)_{j}^{L}\left(G_{s}\right) \\
& \leq \sum_{s=1}^{l} \frac{1}{\min F_{i_{2}}^{\xi}(M)}\left(\sum_{t=2}^{p} \sum_{j=r_{t-1}+1}^{r_{t}}(\xi-1)_{j}^{L}\left(G_{s}\right)\right) \\
& \leq \sum_{s=1}^{l} \frac{1}{\min F_{i_{2}}^{\xi}(M)} \xi \quad \text { by the induction hypothesis } \\
& =\frac{l}{\min F_{i_{2}}^{\xi}(M)} \xi \\
& \leq \xi \quad \operatorname{as} l \leq \min G \leq \max F_{i_{1}}^{\xi}(M)<\min F_{i_{2}}^{\xi}(M) .
\end{aligned}
$$

Finally, $\sum_{t=1}^{p} \xi_{i_{t}}^{M}(G) \leq 1+\xi$ and hence $\sum_{i=1}^{n} \xi_{i}^{M}(G) \leq 1+\xi$. We conclude since $G \in S_{\xi}$ was arbitrary, that $\left\|\sum_{i=1}^{n} \xi_{i}^{M}\right\|_{\xi} \leq \xi+1$, as claimed.

Let now $M \in[\mathbb{N}]$. By identifying elements of $[M]$ with their indicator functions, $[M]$ can be endowed with the topology of pointwise convergence. It is not difficult to see that $[M]$ is then homeomorphic to a $G_{\delta}$ subset of the Cantor set $\{0,1\}^{\mathbb{N}}$, and thus it is a zero-dimensional Polish space. Further, $[M]$ is perfect (that is, contains no isolated points) and every compact subset of $[M]$ is nowhere dense. It is then a classical result that $[M]$, endowed with the topology of pointwise convergence, is homeomorphic to the space of irrational numbers with the ordinary topology. It is worthwhile to note here that the family

$$
\left\{W\left(p_{1}, \ldots, p_{k}\right): k \in \mathbb{N}, p_{1}<\ldots<p_{k}, p_{i} \in M, i \leq k\right\}
$$

where $W\left(p_{1}, \ldots, p_{k}\right)=\left\{L \in[M], L=\left(l_{i}\right): l_{i}=p_{i}, i \leq k\right\}$, forms a basis of clopen subsets for the topology of pointwise convergence in $[M]$.

3. Proofs of the main results. This section is devoted to the proofs of Theorems 1.1 and 1.3 .

Definition 3.1. Let $\xi<\omega_{1}$ and $A \in[\mathbb{N}]<\infty$. We set

$$
\tau_{\xi}(A)=\max \left\{n \in \mathbb{N}: A \cap F_{n}^{\xi}(A \cup\{m \in \mathbb{N}: m>\max A\}) \neq \emptyset\right\} .
$$


We observe that $\tau_{\xi}(A)$ remains invariant if $\{m \in \mathbb{N}: m>\max A\}$ is replaced by $\{m \in M: m>\max A\}, M \in[\mathbb{N}]$, in Definition 3.1. The quantity $\tau_{\xi}(A)$ is important for our purposes since it will enable us to state a criterion for determining whether or not the sequence $\left(e_{l_{n}}^{\xi}\right)$ dominates $\left(e_{m_{n}}^{\xi}\right)$, where $L=\left(l_{n}\right)$ and $M=\left(m_{n}\right)$ belong to $[\mathbb{N}$. Our next lemma describes some permanence properties of $\tau_{\xi}(A)$.

LEMMA 3.2. Let $\xi<\omega_{1}$ and $A, B$ belong to $[\mathbb{N}]^{<\infty}$.

1. If $A \subset B$ then $\tau_{\xi}(A) \leq \tau_{\xi}(B)$.

2. If $A<B$ then $\tau_{\xi}(A \cup B) \leq \tau_{\xi}(A)+\tau_{\xi}(B)$.

3. If $A=\left\{a_{1}<\ldots<a_{n}\right\}, B=\left\{b_{1}<\ldots<b_{n}\right\}, n \in \mathbb{N}$, and $a_{i} \leq b_{i}$ for $i \leq n$, then $\tau_{\xi}(B) \leq \tau_{\xi}(A)$.

4. Assume that $A=\bigcup_{i=1}^{n} A_{i}, B=\bigcup_{i=1}^{n} B_{i}$, where $n \in \mathbb{N}$, and $A_{1}<$ $\ldots<A_{n}, B_{1}<\ldots<B_{n}$ are maximal members of $S_{\xi}$. If $\min A_{i} \leq \min B_{i}$ for all $i \leq n$, then $\tau_{\xi+1}(B) \leq \tau_{\xi+1}(A)$.

5. Assume that $A=\bigcup_{i=1}^{n} A_{i}$ for some $n \in \mathbb{N}$. Then

$$
\tau_{\xi}(A) \leq\left(\sum_{i=1}^{n} \tau_{\xi}\left(A_{i}\right)\right)(\xi+1)+1
$$

for any $\xi<\omega$.

Proof. The first two properties are immediate consequences of Definition 3.1. The third property follows because $S_{\xi}$ is spreading. Let us show that 4 holds. This is accomplished by induction on $n$. The case $n=1$ is easy because $\tau_{\xi+1}(B)=\tau_{\xi+1}(A)=1$. Assuming the assertion true for all $k<n$, we set $k_{1}=\min A_{1}$ and $l_{1}=\min B_{1}$. In case $l_{1} \geq n$, we obtain $B \in S_{\xi+1}$. Thus $\tau_{\xi+1}(B)=1$ and hence the assertion holds.

Next suppose that $l_{1}<n$. It follows that $\bigcup_{i=1}^{k_{1}} A_{i}$ and $\bigcup_{i=1}^{l_{1}} B_{i}$ are maximal $S_{\xi+1}$ sets. On the other hand, because $n-l_{1}<n$, the induction hypothesis yields $\tau_{\xi+1}\left(\bigcup_{i=l_{1}+1}^{n} B_{i}\right) \leq \tau_{\xi+1}\left(\bigcup_{i=l_{1}+1}^{n} A_{i}\right)$. But also $k_{1} \leq l_{1}$, and so property 1 yields that $\tau_{\xi+1}\left(\bigcup_{i=l_{1}+1}^{n} A_{i}\right) \leq \tau_{\xi+1}\left(\bigcup_{i=k_{1}+1}^{n} A_{i}\right)$. The proof is complete since $\tau_{\xi+1}(B)=1+\tau_{\xi+1}\left(\bigcup_{i=l_{1}+1}^{n} B_{i}\right)$, while $\tau_{\xi+1}(A)=$ $1+\tau_{\xi+1}\left(\bigcup_{i=k_{1}+1}^{n} A_{i}\right)$.

We now prove 5 . Let $k=\tau_{\xi}(A)$ and $M=A \cup\{m \in \mathbb{N}: m>\max A\}$. Denote $\sum_{j=1}^{k-1} \xi_{j}^{M}$ by $x$. By Lemma $2.3,\|x\|_{\xi} \leq \xi+1$. Hence

$$
k-1=x(A) \leq \sum_{i=1}^{n} x\left(A_{i}\right) \leq\left(\sum_{i=1}^{n} \tau_{\xi}\left(A_{i}\right)\right)\|x\|_{\xi} \leq\left(\sum_{i=1}^{n} \tau_{\xi}\left(A_{i}\right)\right)(\xi+1),
$$

from which the result follows.

DeFINITION 3.3. Let $\xi<\omega_{1}$ and $L=\left(l_{n}\right), M=\left(m_{n}\right)$ belong to $[\mathbb{N}]$.
Define

$$
d_{\xi}(L, M)=\sup \left\{\tau_{\xi}\left(\phi^{-1} A\right): A \in S_{\xi}[M]\right\},
$$

where $\phi: L \rightarrow M$ is the natural bijection $\phi\left(l_{n}\right)=m_{n}$ for all $n \in \mathbb{N}$.

The reason we introduced the quantity $d_{\xi}(L, M)$ is justified by our next lemma.

LEMMA 3.4. Let $\xi<\omega$ and $L=\left(l_{n}\right), M=\left(m_{n}\right)$ belong to [N]. Then $\left(e_{l_{n}}^{\xi}\right)$ dominates $\left(e_{m_{n}}^{\xi}\right)$ if and only if $d_{\xi}(L, M)$ is finite.

Proof. Suppose first that $d_{\xi}(L, M)=p<\infty$. Let $\left(a_{i}\right)_{i=1}^{n}$ be scalars and choose $F \in S_{\xi}[M]$ so that $\sum_{i \in H}\left|a_{i}\right|=\left\|\sum_{i=1}^{n} a_{i} e_{m_{i}}^{\xi}\right\|$, where we have set $H=\left\{i \leq n: m_{i} \in F\right\}$. It follows, by our assumption, that we can find successive $S_{\xi}[L]$ sets $G_{1}<\ldots<G_{p}$ so that $\phi^{-1} F \subset \bigcup_{j=1}^{p} G_{j}$. We now set $H_{j}=\left\{i \in H: l_{i} \in G_{j}\right\}$ for all $j \leq p$. It is clear that $H=\bigcup_{j=1}^{p} H_{j}$, and moreover, $\left\{l_{i}: i \in H_{j}\right\}$ belongs to $\bar{S}_{\xi}[L]$. Finally,

$$
\sum_{i \in H}\left|a_{i}\right|=\sum_{j=1}^{p} \sum_{i \in H_{j}}\left|a_{i}\right| \leq p\left\|\sum_{i=1}^{n} a_{i} e_{l_{i}}^{\xi}\right\| .
$$

Thus, $\left\|\sum_{i=1}^{n} a_{i} e_{m_{i}}^{\xi}\right\| \leq p\left\|\sum_{i=1}^{n} a_{i} e_{l_{i}}^{\xi}\right\|$.

Conversely, assume that $\left(e_{l_{n}}^{\xi}\right) C$-dominates $\left(e_{m_{n}}^{\xi}\right)$ and let $F \in S_{\xi}[M]$. Suppose that $\tau_{\xi}\left(\phi^{-1} F\right)=k$. It follows that there exist successive maximal $S_{\xi}[L]$ sets $G_{1}<\ldots<G_{k-1}$ so that $\bigcup_{i=1}^{k-1} G_{i} \subset \phi^{-1} F$. Put

$$
Q=\bigcup_{i=1}^{k-1} G_{i} \cup\left\{l \in L: l>\max G_{k-1}\right\} .
$$

We may write $\xi_{i}^{Q}=\sum_{j \in G_{i}} a_{j}^{i} e_{j}^{\xi}$ with $\sum_{j \in G_{i}} a_{j}^{i}=1$ for all $i \leq k-1$. If we apply Lemma 2.3 , we obtain

$$
C(\xi+1) \geq C\left\|\sum_{i=1}^{k-1} \xi_{i}^{Q}\right\|_{\xi}=C\left\|\sum_{i=1}^{k-1} \sum_{j \in G_{i}} a_{j}^{i} e_{j}^{\xi}\right\| \geq\left\|\sum_{i=1}^{k-1} \sum_{j \in G_{i}} a_{j}^{i} e_{\phi(j)}^{\xi}\right\| \geq k-1,
$$

as $\bigcup_{i=1}^{k-1}\left\{\phi(j): j \in G_{i}\right\} \subset F$ and $\sum_{j \in G_{i}} a_{j}^{i}=1$. Hence $k \leq C(\xi+1)+1$ which implies that $d_{\xi}(L, M) \leq C(\xi+1)+1$ as $F$ was an arbitrary $S_{\xi}[M]$ set.

We shall next show that $\left(e_{n}^{\xi}\right)$ has "many" non-equivalent subsequences.

LEMMA 3.5. Let $1 \leq \xi<\omega, N \in[\mathbb{N}]$ and set

$$
\mathcal{D}=\left\{(L, M) \in[N] \times[N]: d_{\xi}(L, M)=d_{\xi}(M, L)=\infty\right\} .
$$

Then $\mathcal{D}$ is a $G_{\delta}$ dense subset of $[N] \times[N]$. 
Proof. By Baire's theorem, it suffices to show that the sets $\{(L, M) \in$ $\left.[N] \times[N]: d_{\xi}(L, M)<\infty\right\}$ and $\left\{(L, M) \in[N] \times[N]: d_{\xi}(M, L)<\infty\right\}$ are first category $F_{\sigma}$ subsets of $[N] \times[N]$. Indeed, we may write

$$
\left\{(L, M): d_{\xi}(L, M)<\infty\right\}=\bigcup_{n=1}^{\infty}\left\{(L, M): d_{\xi}(L, M) \leq n\right\} .
$$

It is easy to see that each set in the union is closed in $[N] \times[N]$ and thus it remains to show that $\left\{(L, M) \in[N] \times[N]: d_{\xi}(L, M) \leq n\right\}$ has empty interior in $[N] \times[N]$. If that were not the case, choose non-empty basic clopen subsets $\mathcal{U}$ and $\mathcal{V}$ of $[N]$ so that $\mathcal{U} \times \mathcal{V}$ is contained in $\{(L, M) \in[N] \times[N]$ : $\left.d_{\xi}(L, M) \leq n\right\}$. There exist $p_{1}<\ldots<p_{k}$ in $N$ so that $\mathcal{V}=W\left(p_{1}, \ldots, p_{k}\right)$. Fix $L \in \mathcal{U}, L=\left(l_{i}\right)$. If $M \in[N]$ with $\min M>p_{k}$, let $P=\left\{p_{1}, \ldots, p_{k}\right\} \cup M$. Since $d_{\xi}(L, P) \leq n$, it follows that if $L_{k}=\left(l_{k+i}\right)_{i=1}^{\infty}$, then $d_{\xi}\left(L_{k}, M\right) \leq n$. By Lemma 3.4 this implies that $\left(e_{l}^{\xi}\right)_{l \in L_{k}}$ is equivalent to the unit vector basis of $\ell_{1}$, which is absurd. Arguing similarly, we also find that $\{(L, M) \in$ $\left.[N] \times[N]: d_{\xi}(M, L)<\infty\right\}$ is a first category $F_{\sigma}$ subset of $[N] \times[N]$.

We also need the following result which is a special case of a theorem by Mycielski [12] and Kuratowski [10] (cf. also [9]).

Proposimion 3.6. Let $K$ be a perfect Polish space and $G$ a $G_{\delta}$ dense subset of $K \times K$. There exists a subset $C$ of $K$ homeomorphic to the Cantor set such that $C \times C \backslash \Delta \subset G$ (here $\Delta$ is the diagonal of $K \times K$ ).

This result may be found in [9] (p. 129, Theorem 19.1) but we shall include a proof to be thorough.

LEMMA 3.7. Let $K$ be Polish and $G$ be an open dense subset of $K \times K$. Let $\left(A_{i}\right)_{i=1}^{n}(n \geq 2)$ be a finite sequence of open non-empty subsets of $K$. Then for every $\varepsilon>0$ there exist open non-empty subsets $\left(B_{i}\right)_{i=1}^{n}$ of $K$ so that $\operatorname{diam} B_{i}<\varepsilon$ for all $i \leq n$ and $\bar{B}_{i} \times \bar{B}_{j} \subset G \cap\left(A_{i} \times A_{j}\right)$ for all $i \neq j$ in $\{1, \ldots, n\}$.

Proof. By induction on $n$. Suppose first that $n=2$. Since $\left(A_{1} \times A_{2}\right) \cap$ $G \neq \emptyset$, there exist open non-empty subsets $C_{1}, C_{2}$ of $K$, with diameters smaller than $\varepsilon$, so that $\bar{C}_{1} \times \bar{C}_{2} \subset G \cap\left(A_{1} \times A_{2}\right)$. Further, $\left(C_{2} \times C_{1}\right) \cap G \neq \emptyset$ and thus there exist open non-empty subsets $B_{1}, B_{2}$ of $K$ so that $\bar{B}_{2} \times \bar{B}_{1} \subset$ $G \cap\left(C_{2} \times C_{1}\right)$. Of course, $B_{1}$ and $B_{2}$ satisfy the conclusion of the lemma for $n=2$.

Next assume $n>2$ and that the result holds for $n-1$. We can therefore choose open non-empty subsets $\left(C_{i}\right)_{i=1}^{n-1}$ of $K$, with diameters smaller than $\varepsilon$, so that $\bar{C}_{i} \times \bar{C}_{j} \subset G \cap\left(A_{i} \times A_{j}\right)$, for all $i \neq j$ in $\{1, \ldots, n-1\}$. Next, set $A_{n, 0}=A_{n}$ and choose, as in the case $n=2$, non-empty open subsets $\left(B_{i}\right)_{i=1}^{n-1},\left(A_{n, i}\right)_{i=1}^{n-1}$ of $K$, with diameters smaller than $\varepsilon$, so that $\bar{B}_{i} \times \bar{A}_{n, i} \subset$
$G \cap\left(C_{i} \times A_{n, i-1}\right)$ and $\bar{A}_{n, i} \times \bar{B}_{i} \subset G \cap\left(A_{n, i-1} \times C_{i}\right)$, for all $i \leq n-1$. Set $B_{n}=A_{n, n-1}$ and it is easy to check that $\left(B_{i}\right)_{i=1}^{n}$ is the desired sequence.

Proof of Proposition 3.6. Since $K$ contains no isolated points, $\Delta$ is nowhere dense in $K \times K$. Hence, $G \cap(K \times K \backslash \Delta)$ is a $G_{\delta}$ dense subset of $K \times K$. We shall therefore assume, without loss of generality, that $G \cap \Delta=\emptyset$. Now let $\left(G_{n}\right)$ be a decreasing sequence of open dense subsets of $K \times K$ whose intersection is $G$. We can assume that $G_{n} \cap \Delta=\emptyset$ for all $n \in \mathbb{N}$.

We shall construct a collection $\left\{U_{\alpha}: \alpha \in\{0,1\}^{n}, n \in \mathbb{N}\right\}$ of open nonempty subsets of $K$ so that the following properties are satisfied for every $n \in \mathbb{N}$ :

(i) $\bar{U}_{\alpha} \cap \bar{U}_{\beta}=\emptyset$ whenever $\alpha \neq \beta$ in $\{0,1\}^{n}$.

(ii) $\bar{U}_{\alpha} \subset U_{\beta}$ for all $\alpha \in\{0,1\}^{n}$ and every $\beta \in\{0,1\}^{m}(m<n)$ which is an initial segment of $\alpha$.

(iii) $\operatorname{diam} U_{\alpha}<1 / n$ for every $\alpha \in\{0,1\}^{n}$

(iv) $\bar{U}_{\alpha} \times \bar{U}_{\beta} \subset G_{n}$ whenever $\alpha \neq \beta$ in $\{0,1\}^{n}$.

Once this is accomplished, we let

$$
C=\left\{x \in K: \exists \alpha \in\{0,1\}^{\mathbb{N}},\{x\}=\bigcap_{n=1}^{\infty} U_{\alpha \mid n}\right\},
$$

where $\alpha \mid n=\left(a_{1}, \ldots, a_{n}\right)$ if $\alpha=\left(a_{i}\right) \in\{0,1\}^{\mathbb{N}}$. It is a standard result that $C$ is homeomorphic to the Cantor set. Property (iv) yields that $C$ satisfies the conclusion of Proposition 3.6.

The construction is done by induction on $n \in \mathbb{N}$. For $n=1$ choose open non-empty subsets $W_{0}$ and $W_{1}$ of $K$ so that $W_{0} \times W_{1} \subset G_{1}$. Then $W_{0}$ and $W_{1}$ are disjoint since $G_{1} \cap \Delta=\emptyset$. If we apply Lemma 3.7, for $\varepsilon=1$, to the dense open subset $G_{1}$ and the open sets $W_{0}$ and $W_{1}$, we shall find non-empty open subsets $U_{0}, U_{1}$ of $K$ having properties (i)-(iv) for $n=1$.

Now suppose that for every $k \leq n$ we have constructed a collection $\left\{U_{\alpha}: \alpha \in\{0,1\}^{k}\right\}$ of open non-empty subsets of $K$ whose members share properties (i)-(iv) for $k$. Let $\left\{d_{1}, \ldots, d_{p}\right\}, p=2^{n}$, be an enumeration of $\{0,1\}^{n}$. Another application of Lemma 3.7 yields non-empty open subsets $W_{j 0}$ and $W_{j 1}$ of $K, j \leq p$, so that $\bar{W}_{j r} \times \bar{W}_{j s} \subset\left(U_{d_{j}} \times U_{d_{j}}\right) \cap G_{n+1}$ for every $j \leq p$ and all pairs $(r, s)$ of distinct elements of $\{0,1\}$. It follows, since $G_{n+1} \cap \Delta=\emptyset$, that $W_{j 0} \cap W_{j 1}=\emptyset$. According to the induction hypothesis $\bar{U}_{d_{j}} \cap \bar{U}_{d_{i}}=\emptyset$ for all $i \neq j$ in $\{1, \ldots, p\}$, and thus $\bar{W}_{j r} \cap \bar{W}_{i s}=\emptyset$ for all $(j, r) \neq(i, s)$ in $\{1, \ldots, p\} \times\{0,1\}$.

We next apply Lemma 3.7 , for $\varepsilon=1 /(n+1)$, to the family $\left\{W_{j r}\right.$ : $(j, r) \in\{1, \ldots, p\} \times\{0,1\}\}$ and the dense open subset $G_{n+1}$. We obtain a collection $\left\{U_{\alpha}: \alpha \in\{0,1\}^{n+1}\right\}$ of non-empty open subsets of $K$ so that $\bar{U}_{\alpha} \times \bar{U}_{\beta} \subset\left(W_{j r} \times W_{i s}\right) \cap G_{n+1}$ whenever $\alpha=\left(d_{j}, r\right), \beta=\left(d_{i}, s\right)$ and $(j, r) \neq(i, s)$ in $\{1, \ldots, p\} \times\{0,1\}$. Evidently, $\left\{U_{\alpha}: \alpha \in\{0,1\}^{n+1}\right\}$ has 
properties (i)-(iv). The inductive step and hence the proof of the proposition are now complete.

Assuming we have proved Theorem 1.1 and Corollary 1.2, let us now show how to derive Theorem 1.3 from our previous results.

Proof of Theorem 1.3. Let $\mathcal{D}$ be as in the statement of Lemma 3.5, where we have taken $N=\mathbb{N}$. We can apply Proposition 3.6 for the space $[\mathbb{N}]$ and the set $\mathcal{D}$ to obtain $\mathcal{A} \subset[\mathbb{N}$ homeomorphic to the Cantor set and such that $\mathcal{A} \times \mathcal{A} \backslash \Delta \subset \mathcal{D}$. Lemma 3.4 and Corollary 1.2 yield that $\mathcal{A}$ is the desired subset of $[\mathbb{N}]$.

We shall next pass to the proof of Theorem 1.1. We start with some necessary lemmas.

LEMMA 3.8. Let $G \in[\mathbb{N}]^{<\infty}$ and $\xi<\omega$. The following are equivalent:

1. $G$ is a member (resp. maximal member) of $S_{\xi}$.

2. For every $0 \leq \zeta \leq \xi$ there exist $n \in \mathbb{N}$ and successive members (resp. maximal members) $G_{1}<\ldots<G_{n}$ of $S_{\zeta}$ so that $G=\bigcup_{i=1}^{n} G_{i}$ and $\left\{\min G_{i}\right.$ : $i \leq n\}$ is a member (resp. maximal member) of $S_{\xi-\zeta}$.

3. There exist $0 \leq \zeta \leq \xi, n \in \mathbb{N}$ and successive members (resp. maximal members) $G_{1}<\ldots<G_{n}$ of $S_{\zeta}$ so that $G=\bigcup_{i=1}^{n} G_{i}$ and $\left\{\min G_{i}: i \leq n\right\}$ is a member (resp. maximal member) of $S_{\xi-\zeta}$. of $S_{\xi}$.

roof. We show that all three conditions are equivalent for the members

$1 \Rightarrow 2$. Induction on $\xi$. If $\xi=0$ the assertion is trivial. Suppose now $\xi \geq 1$ and that the assertion holds for $\xi-1$. Let $\zeta \leq \xi$. If $\zeta=\xi$, the assertion is again trivial. So assume $\zeta<\xi$. Choose $H_{1}<\ldots<H_{p}$ in $S_{\xi-1}$ so that $p \leq \min H_{1}$ and $G=\bigcup_{i=1}^{p} H_{i}$. The induction hypothesis yields that for each $i \leq p$ there exist $H_{i 1}<\ldots<H_{i r_{i}}$ in $S_{\zeta}$ so that $\left\{\min H_{i j}\right.$ : $\left.j \leq r_{i}\right\}$ belongs to $S_{\xi-\zeta-1}$ and $H_{i}=\bigcup_{j=1}^{r_{i}} H_{i j}$. Let $\left\{G_{1}, \ldots, G_{n}\right\}$ be an enumeration of the set $\left\{H_{i j}: j \leq r_{i}, i \leq p\right\}$ so that $G_{1}<\ldots<G_{n}$. Note that $\left\{\min G_{i}: i \leq n\right\}=\bigcup_{i=1}^{p}\left\{\min H_{i j}: j \leq r_{i}\right\}$ and so it is a member of $S_{\xi-\zeta}$ as $p \leq \min H_{11}=\min H_{1}$

$2 \Rightarrow 3$. This implication is trivial.

$3 \Rightarrow 1$. Again induction on $\xi$. If $\xi=0$ the assertion is trivial. Suppose now $\xi \geq 1$ and that the assertion holds for $\xi-1$. Let $\zeta \leq \xi$. If $\zeta=\xi$, the assertion is again trivial. So assume $\zeta<\xi$. We first apply $1 \Rightarrow 2$ for the set $\left\{\min G_{i}: i \leq n\right\} \in S_{\xi-\zeta}$ to obtain $H_{1}<\ldots<H_{p}$ in $S_{\xi-\zeta-1}$ so that $\left\{\min G_{i}: i \leq n\right\}=\bigcup_{i=1}^{p} H_{i}$ and $\left\{\min H_{i}: i \leq p\right\} \in S_{1}$. Set $I_{i}=\left\{j \leq n: \min G_{j} \in H_{i}\right\}, i \leq p$. Then $\bigcup_{j \in I_{i}} G_{j} \in S_{\xi-1}$ by the induction hypothesis since $\left\{\min G_{j}: j \in I_{i}\right\}=H_{i}$, which belongs to $S_{\xi-\zeta-1}$. Finally $G=\bigcup_{i=1}^{p}\left(\bigcup_{j \in I_{i}} G_{j}\right) \in S_{\xi}$, as $\min G=\min H_{1} \geq p$. The latter inequality holds because $\left\{\min H_{i}: i \leq p\right\} \in S_{1}$
The proof for the case of maximal Schreier sets requires only minor modifications. Namely, all the sets which belong to an appropriate class $S_{\alpha}$, $\alpha \leq \xi$, and appear in the previous arguments, can be taken to be maximal members of $S_{\alpha}$.

LEMMA 3.9. Let $\xi<\omega_{1}$. Suppose that $L=\left(l_{i}\right), M=\left(m_{i}\right)$ belong to $[\mathbb{N}]$ and satisfy $l_{i}<m_{i}<l_{i+1}$ for every $i \in \mathbb{N}$. Then $\left(e_{l_{i}}^{\xi}\right)$ is 2-equivalent to $\left(e_{m_{i}}^{\xi}\right)$. That is, $\left\|\sum_{i=1}^{n} a_{i} e_{l_{i}}^{\xi}\right\| \leq\left\|\sum_{i=1}^{n} a_{i} e_{m_{i}}^{\xi}\right\| \leq 2\left\|\sum_{i=1}^{n} a_{i} e_{l_{i}}^{\xi}\right\|$ for every $n \in \mathbb{N}$ and all scalar sequences $\left(a_{i}\right)_{i=1}^{n}$.

We omit the easy proof and pass to

LEMMA 3.10. Let $\xi<\omega$ and $0 \leq \zeta \leq \xi$. Let $L \in[\mathbb{N}]$ and set $q_{n}=$ $\min F_{n}^{\zeta}(L)$ for all $n \in \mathbb{N}$. Then $\left(\zeta_{n}^{L}\right)$, considered as a sequence in $X^{\xi}$, is $12(\zeta+1)$-equivalent to $\left(e_{q_{n}}^{\xi-\zeta}\right)$.

Proof. Let $n \in \mathbb{N}$ and $\left(a_{i}\right)_{i=1}^{n}$ be scalars. Choose $G \subset\left\{q_{1}, \ldots, q_{n}\right\}, G \in$ $S_{\xi-\zeta}$, such that $\sum_{i \in I}\left|a_{i}\right|=\left\|\sum_{i=1}^{n} a_{i} e_{q_{i}}^{\xi-\zeta}\right\|$, where $I=\left\{i \leq n: q_{i} \in G\right\}$. It follows that $H=\bigcup_{i \in I} F_{i}^{\zeta}(L) \in S_{\xi}$, by Lemma 3.8. Hence, $\left\|\sum_{i=1}^{n} a_{i} \zeta_{i}^{L}\right\|_{\xi} \geq$ $\sum_{i \in I}\left|a_{i}\right|$ and thus $\left\|\sum_{i=1}^{n} a_{i} e_{q_{i}}^{\xi-\zeta}\right\| \leq\left\|\sum_{i=1}^{n} a_{i} \zeta_{i}^{L}\right\|_{\xi}$.

We next show that $\left\|\sum_{i=1}^{n} a_{i} \zeta_{i}^{L}\right\|_{\xi} \leq 12(\zeta+1)\left\|\sum_{i=1}^{n} a_{i} e_{q_{i}}^{\xi-\zeta}\right\|$. Let $G \in$ $S_{\xi}[L]$ be maximal and put $G_{i}=F_{i}^{\zeta}(L) \cap G$ for all $i \leq n$. We apply Lemma 3.8 to find $p \in \mathbb{N}$ and maximal members $H_{1}<\ldots<H_{p}$ of $S_{\zeta}$ so that $G=$ $\bigcup_{j=1}^{p} H_{j}$ and $\left\{\min H_{j}: j \leq p\right\}$ is a maximal member of $S_{\xi-\zeta}$

We claim that each of the $G_{i}$ 's can intersect at most two of the $H_{j}$ 's. Indeed, assume that for some $i$ and $j_{1}<j_{2}<j_{3}$ we had $G_{i} \cap H_{j_{r}} \neq \emptyset$ for all $r \leq 3$. Then $H_{j_{2}} \subset G_{i}$ because $H_{j_{2}} \subset\left[\min G_{i}, \max G_{i}\right]$. Thus, $H_{j_{2}} \subset F_{i}^{\zeta}(L)$ and hence $H_{j_{2}}=F_{i}^{\zeta}(L)$, by the maximality of $H_{j_{2}}$. It follows that $H_{j_{2}}=$ $G_{i}$, which is a contradiction as $H_{j_{1}} \cap H_{j_{2}}=\emptyset$. Therefore our claim holds and evidently, for each $i \leq n, G_{i}$ intersects either exactly one of the $H_{j}$ 's, or exactly two (consecutive) $H_{j}$ 's. We can thus partition $\{1, \ldots, n\}$ in the following two subsets:

$$
\begin{aligned}
& I_{1}=\left\{i \leq n: G_{i} \subset H_{j} \text { for some } j \leq p\right\}, \\
& I_{2}=\left\{i \leq n: \exists j_{1}<j_{2} \leq p, G_{i} \subset H_{j_{1}} \cup H_{j_{2}}, G_{i} \cap H_{j_{r}} \neq \emptyset, r \leq 2\right\} .
\end{aligned}
$$

Let $k_{i}=\max F_{i}^{\zeta}(L)$ for all $i \leq n$. We now need the following

CLAIM. Suppose that $T_{i} \subset F_{i}^{\zeta}(L)$ for all $i \leq n$. Assume also that for each $i \leq n$ there exists $j \leq p$ so that $T_{i} \subset H_{j}$. Then

$$
\left|\sum_{i=1}^{n} a_{i} \zeta_{i}^{L}\left(\bigcup_{m=1}^{n} T_{m}\right)\right| \leq(\zeta+1)\left\|\sum_{i=1}^{n} a_{i} e_{k_{i}}^{\xi-\zeta}\right\|
$$


Once the claim is established we finish the proof as follows: Observe that our claim yields

$$
\left|\sum_{i \in I_{1}} a_{i} \zeta_{i}^{L}(G)\right| \leq(\zeta+1)|| \sum_{i=1}^{n} a_{i} e_{k_{i}}^{\xi-\zeta} \mid .
$$

On the other hand, if $i \in I_{2}$ there exist $A_{i}<B_{i}$ so that $G_{i}=A_{i} \cup B_{i}$ and each element of $\left\{A_{i}, B_{i}\right\}$ is contained in some $H_{j}$. Our claim then yields that

$$
\left|\sum_{i \in I_{2}} a_{i} \zeta_{i}^{L}(G)\right| \leq 2(\zeta+1) \| \sum_{i=1}^{n} a_{i} e_{k_{i}}^{\xi-\zeta}|| .
$$

Therefore, $\left|\sum_{i=1}^{n} a_{i} \zeta_{i}^{L}(G)\right| \leq 3(\zeta+1)\left\|\sum_{i=1}^{n} a_{i} e_{k_{i}}^{\xi-\zeta}\right\|$. It now follows, since $G$ was arbitrary, that $\left\|\sum_{i=1}^{n} a_{i} \zeta_{i}^{L}\right\|_{\xi} \leq 6(\zeta+1)\left\|\sum_{i=1}^{n} a_{i} e_{k_{i}}^{\xi-\zeta}\right\|$. The desired estimate now follows from Lemma 3.9.

We proceed to prove our claim. Let $R_{j}=\left\{i \leq n: T_{i} \neq \emptyset, T_{i} \subset H_{j}\right\}$, $j \leq p$, and choose $i_{j} \in R_{j}$ such that $\max _{r \in R_{j}}\left|a_{r}\right|=\left|a_{i_{j}}\right|$. Then

$$
\begin{aligned}
\left|\sum_{i=1}^{n} a_{i} \zeta_{i}^{L}\left(\bigcup_{m=1}^{n} T_{m}\right)\right| & =\left|\sum_{i=1}^{n} a_{i} \zeta_{i}^{L}\left(T_{i}\right)\right|=\left|\sum_{j=1}^{p} \sum_{i \in R_{j}} a_{i} \zeta_{i}^{L}\left(T_{i}\right)\right| \\
& \leq \sum_{j=1}^{p}\left|\left(\sum_{i \in R_{j}} a_{i} \zeta_{i}^{L}\right)\left(\bigcup_{m \in R_{j}} T_{m}\right)\right| \\
& \leq \sum_{j=1}^{p}\left\|\mid \sum_{i \in R_{j}} a_{i} \zeta_{i}^{L}\right\|_{\zeta_{i}} \quad \text { because } \bigcup_{m \in R_{j}} T_{m} \in S_{\zeta} \\
& \leq \sum_{j=1}^{p}(\zeta+1) \max _{i \in R_{j}}\left|a_{i}\right| \quad \text { by Lemma } 2.3 \\
& =\sum_{j=1}^{p}(\zeta+1)\left|a_{i_{j}}\right| \leq(\zeta+1) \| \sum_{i=1}^{n} a_{i} e_{k_{i}}^{\xi^{--\zeta} \| .}
\end{aligned}
$$

The last inequality holds since $T_{i_{j}} \subset H_{j}$ implies that $\min H_{j} \leq k_{i_{j}}$ for all $j \leq p$ and thus $\left\{k_{i_{j}}: j \leq p\right\} \in S_{\xi-\zeta}$.

We recall here that a bounded sequence $\left(x_{n}\right)$ in some Banach space is said to be an $\ell_{1}^{\xi}$-spreading model, $\xi<\omega_{1}$, provided that there exists a constant $C>0$ so that $\left\|\sum_{i \in F} a_{i} x_{i}\right\| \geq C \sum_{i \in F}\left|a_{i}\right|$ for every $F \in S_{\xi}$ and all scalars $\left(a_{i}\right)_{i \in F}$.

REMARK. It is easy to see that every subsequence of $\left(e_{n}^{\xi}\right)$ is an $\ell_{1}^{\xi}$-spreading model in $X^{\xi}$. However, Lemma 2.3 implies that no subsequence of $\left(e_{n}^{\xi}\right)$ is an $\ell_{1}^{\xi+1}$-spreading model in $X^{\xi}$.
Proposition 3.11. Suppose $L=\left(l_{i}\right), M=\left(m_{i}\right)$ belong to $[\mathbb{N}]$ and that $\xi<\omega$. Assume further that there exist a map $\psi: L \rightarrow M$ and $a$ bounded linear operator $T: X_{L}^{\xi} \rightarrow X_{M}^{\xi}$ so that $T\left(e_{l}^{\xi}\right)=e_{\psi(l)}^{\xi}$ for every $l \in L$. Then there exists an integer $D>0$ so that $\tau_{\zeta}\left(\psi^{-1} F\right) \leq D$ for every $F \in S_{\zeta}[M]$ and all $0 \leq \zeta \leq \xi$.

Proof. We first note that $\psi^{-1} F \in[L]^{<\infty}$ for every $F \in[M]^{<\infty}$. Indeed, if that were not the case, we would find $m \in M$ and $N \in[L]$ so that $\psi(l)=m$ for every $l \in N$. It follows that $T\left(e_{l}^{\xi}\right)=e_{m}^{\xi}$ for all $l \in N$, contradicting our assumption that $T$ is bounded.

Fix $0 \leq \zeta<\xi$. Our first task is to show that $\sup _{n} \tau_{\zeta}\left(\psi^{-1} F_{n}^{\zeta}(P)\right)<\infty$ for every $P \in[M]$. Suppose instead that $\sup _{n} \tau_{\zeta}\left(\psi^{-1} F_{n}^{\zeta}(P)\right)=\infty$ for some $P \in[M]$. We claim that there exist a sequence of positive integers, $\left(n_{i}\right)$, and a sequence of successive maximal $S_{\zeta+1}[L]$ sets, $\left(G_{i}\right)$, so that letting $q_{i}=\min G_{i}$ for all $i \in \mathbb{N}$ we get

$$
G_{i} \backslash\left\{q_{i}\right\} \subset \psi^{-1} F_{n_{i}}^{\zeta}(P) \text { for all } i \in \mathbb{N} .
$$

Indeed, choose $n_{1}$ so that $\tau_{\zeta}\left(\psi^{-1} F_{n_{1}}^{\zeta}(P)\right)>l_{1}$. Put $q_{1}=l_{1}$. Because $\psi^{-1} F_{n_{1}}^{\zeta}(P)$ contains at least $l_{1}$ successive maximal $S_{\zeta}[L]$ sets, it is clear that there exists $H_{1} \subset \psi^{-1} F_{n_{1}}^{\zeta}(P), q_{1}<\min H_{1}$, so that $G_{1}=\left\{q_{1}\right\} \cup H_{1}$ is a maximal $S_{\zeta+1}[L]$ set.

Put $l_{t_{1}}=\max G_{1}$ and $w_{1}=\tau_{\zeta}\left(\left\{l_{1}, \ldots, l_{t_{1}}\right\}\right)$. We can find $n_{2}>n_{1}$ so that $\tau_{\zeta}\left(\psi^{-1} F_{n_{2}}^{\zeta}(P)\right)>w_{1}+l_{t_{1}+1}$. Now, $\left\{l \in L: l \geq l_{t_{1}+1}\right\} \cap \psi^{-1} F_{n_{2}}^{\zeta}(P)$ must contain at least $l_{t_{1}+1}$ successive maximal $S_{\zeta}[L]$ sets. If not, then $\tau_{\zeta}[\{l \in L$ : $\left.\left.l \geq l_{t_{1}+1}\right\} \cap \psi^{-1} F_{n_{2}}^{\zeta}(P)\right] \leq l_{t_{1}+1}$ and thus $\tau_{\zeta}\left(\psi^{-1} F_{n_{2}}^{\zeta}(P)\right) \leq l_{t_{1}+1}+w_{1}$, by Lemma 3.2. But this contradicts the choice of $n_{2}$.

We set $q_{2}=l_{t_{1}+1}$ and arguing as we did in the case $i=1$, we can find $H_{2} \subset \psi^{-1} F_{n_{2}}^{\zeta}(P), q_{2}<\min H_{2}$, so that $G_{2}=\left\{q_{2}\right\} \cup H_{2}$ is a maximal $S_{\zeta+1}[L]$ set. We next put $l_{t_{2}}=\max G_{2}$ and continue in the same fashion to obtain sequences $\left(n_{i}\right),\left(G_{i}\right)$ with the desired properties.

Let $Q=\bigcup_{i=1}^{\infty} G_{i}$. Clearly, $Q \in[L]$ and $F_{i}^{\zeta+1}(Q)=G_{i}$ for all $i \in \mathbb{N}$. We now set $R=\bigcup_{i=1}^{\infty} F_{n_{i}}^{\zeta}(P)$. Then $R \in[M]$ and $F_{i}^{\zeta}(R)=F_{n_{i}}^{\zeta}(P)$ for all $i \in \mathbb{N}$. We observe that if $q \in G_{i} \backslash\left\{q_{i}\right\}$, then $T\left(e_{q}^{\xi}\right)=e_{m}^{\xi}$ for some $m \in F_{i}^{\zeta}(R)$.

Next write $(\zeta+1)_{i}^{Q}=a_{i} e_{q_{i}}^{\xi}+\left(1-a_{i}\right) u_{i}$ for all $i \in \mathbb{N}$. Here, $u_{i}$ is a convex combination of the vectors $\left(e_{q}^{\xi}\right)_{q \in G_{i} \backslash\left\{q_{i}\right\}}$ and $0<a_{i}<1$. Evidently, $\lim _{i} a_{i}$ $=0$. Observe that $T u_{i}$ is a convex combination of the vectors $\left(e_{m}^{\xi}\right)_{m \in F_{i}^{\zeta}(R)}$ and thus $\left\|T u_{i}\right\|_{\xi}=1$ for all $i \in \mathbb{N}$.

It must be the case that $\zeta<\xi$ for if not, Lemma 2.3 yields $\lim _{i}\left\|u_{i}\right\|_{\xi}=0$. On the other hand $\left\|T u_{i}\right\|_{\xi}=1$ for all $i \in \mathbb{N}$. Hence $T$ is not bounded, contrary to our assumption. Therefore, $\zeta<\xi$ and so $\left\|u_{i}\right\|_{\xi}=1$ for all $i \in \mathbb{N}$. 
Recall that $\lim _{i}\left\|\left(1-a_{i}\right) T u_{i}\right\|_{\xi}=1$ and $\left(1-a_{i}\right) T u_{i}$ is supported by $F_{i}^{\zeta}(R)$. Using Lemma 3.8 , it is easy to check that $\left(\left(1-a_{i}\right) T u_{i}\right)$ is an $\ell_{1}^{\xi-\zeta}$-spreading model in $X_{M}^{\xi}$, and consequently, since $\lim _{i} a_{i}=0$, also $\left(T\left[(\zeta+1)_{i}^{Q}\right]\right)$ is an $\ell_{1}^{\xi-\zeta}$-spreading model in $X_{M}^{\xi}$. We conclude, as $T$ is bounded, that $\left((\zeta+1)_{i}^{Q}\right)$ is an $\ell_{1}^{\xi-\zeta}$-spreading model in $X^{\xi}$. However, if we apply Lemma 3.10 we deduce that $\left(e_{q_{n}}^{\xi-\zeta-1}\right)$ is an $\ell_{1}^{\xi-\zeta}$-spreading model in $X^{\xi-\zeta-1}$. But this contradicts the Remark after Lemma 3.10. Hence, $\sup _{n} \tau_{\zeta}\left(\psi^{-1} F_{n}^{\zeta}(P)\right)<\infty$ for every $P \in[M]$. It follows that

$$
[M]=\bigcup_{k=1}^{\infty}\left\{P \in[M]: \tau_{\zeta}\left(\psi^{-1} F_{n}^{\zeta}(P)\right) \leq k, \forall n \in \mathbb{N}\right\} .
$$

It is easily seen that every set in the union is closed in $[M]$. Baire's theorem now yields $k_{\zeta} \in \mathbb{N}$ and $r_{1}^{\zeta}<\ldots<r_{s_{\zeta}}^{\zeta}$ in $M$ so that if $P \in[M], P=\left(p_{i}\right)$, and $p_{i}=r_{i}^{\zeta}, i \leq s_{\zeta}$, then $\tau_{\zeta}\left(\psi^{-1} F_{n}^{\zeta}(P)\right) \leq k_{\zeta}$ for all $n \in \mathbb{N}$. It now follows that there exists $m_{0}^{\zeta} \in M$ so that if $F \in S_{\zeta}[M], \min F>m_{0}^{\zeta}$, then $\tau_{\zeta}\left(\psi^{-1} F\right) \leq k_{\zeta}$.

Finally, choose $D_{\zeta} \in \mathbb{N}$ so that $\tau_{\zeta}\left(\psi^{-1} F\right) \leq D_{\zeta}$ for every $F \in S_{\zeta}[M]$, $\max F \leq m_{0}^{\zeta}$. Part 5 of Lemma 3.2 now yields $\tau_{\zeta}\left(\psi^{-1} F\right) \leq\left(D_{\zeta}+k_{\zeta}\right)(\zeta+$ 1) +1 for every $F \in S_{\zeta}[M]$. To complete the proof we need only take $D=\max \left\{\left(D_{\zeta}+k_{\zeta}\right)(\zeta+1)+1: \zeta \leq \xi\right\}$.

PRoposition 3.12. Suppose $L=\left(l_{i}\right), M=\left(m_{i}\right)$ belong to $[\mathbb{N}]$ and that $\xi<\omega$. Assume further that there exist a map $\psi: L \rightarrow M$ and an integer $D>0$ so that $\tau_{\zeta}\left(\psi^{-1} F\right) \leq D$ for every $F \in S_{\zeta}[M]$ and all $0 \leq \zeta \leq \xi$. Then there exist integer constants $E_{\zeta}, 0 \leq \zeta \leq \xi$, so that $\tau_{\zeta}\left(\phi^{-1} F\right) \leq E_{\zeta}$ for every $F \in S_{\zeta}[M]$ and all $0 \leq \zeta \leq \xi(\phi: L \rightarrow M$ is the natural bijection $\left.\phi\left(l_{i}\right)=m_{i}\right)$.

Proof. If $\zeta=0$ it is trivial $\left(E_{0}=1\right)$. Suppose the assertion holds for some $\zeta \leq \xi-1$. We show $E_{\zeta+1}=\left[(\zeta+1) E_{\zeta}+1\right][(2 D+1)(\zeta+2)+1]$ works for $\zeta+1$. Let $F \in S_{\zeta+1}[M], F=\left\{m_{i_{1}}, \ldots, m_{i_{p}}\right\}$. Our hypothesis yields that $\left\{l_{i_{1}}, \ldots, l_{i_{p}}\right\}$ is contained in the union of $E_{\zeta} m_{i_{1}} S_{\zeta}[L]$ sets and so $\tau_{\zeta}\left(\left\{l_{i_{1}}, \ldots, l_{i_{p}}\right\}\right) \leq(\zeta+1) E_{\zeta} m_{i_{1}}+1$ by part 5 of Lemma 3.2. Choose $q_{1} \in \mathbb{N}$ so that the set $\left\{l_{i_{1}+j}: 0 \leq j \leq q_{1}\right\}$ is the union of exactly $\left[(\zeta+1) E_{\zeta}+1\right] m_{i_{1}}$ successive maximal $S_{\zeta}[L]$ sets.

Crarm. $\tau_{\zeta+1}\left(\left\{l_{i_{1}+j}: 0 \leq j \leq q_{1}\right\}\right) \leq E_{\zeta+1}$.

Once our claim is proven, we apply Lemmas 2.2 and 3.2 (parts 4 and 1 ) to conclude that $\tau_{\zeta+1}\left(\left\{l_{i_{1}+j}: 0 \leq j \leq p-1\right\}\right) \leq E_{\zeta+1}$ and $\tau_{\zeta+1}\left(\left\{l_{i_{1}}, \ldots, l_{i_{p}}\right\}\right)$ $\leq E_{\zeta+1}$.

To prove the claim we choose $q<q_{1}$ so that the set $\left\{l_{i_{1}+j}: 0 \leq j \leq q\right\}$ is the union of exactly $m_{i_{1}}$ successive maximal $S_{\zeta}[L]$ sets. Our task now is to show that $\tau_{\zeta+1}\left(\left\{l_{i_{1}+j}: 0 \leq j \leq q\right\}\right) \leq(2 D+1)(\zeta+2)+1$. The claim will then follow by applying parts 4 and 2 of Lemma 3.2.

We first observe that if $0 \leq j_{0} \leq q$ is chosen so that $l_{i_{1}+j} \leq m_{i_{1}+j}$ for all $j \leq j_{0}$, then $\left\{l_{i_{1}+j}: 0 \leq j \leq j_{0}\right\}$ is contained in the union of $2 D S_{\zeta+1}[L]$ sets.

Indeed, $\left\{m_{i_{1}+j}: 0 \leq j \leq j_{0}\right\}$ belongs to $S_{\zeta+1}[M]$, by part 3 of Lemma 3.2 and the fact that $\tau_{\zeta}\left(\left\{l_{i_{1}+j}: 0 \leq j \leq j_{0}\right\} \leq m_{i_{1}}\right.$. It now follows that $\Psi=$ $\left\{\psi\left(l_{i_{1}+j}\right): 0 \leq j \leq j_{0}, \psi\left(l_{i_{1}+j}\right) \geq m_{i_{1}}\right\}$ belongs to $S_{\zeta+1}[M]$. To see this let $\left\{m_{t_{0}}<\ldots<m_{t_{k}}\right\}$, where $k \leq j_{0}$ and $i_{1} \leq t_{0}$, be an enumeration of $\Psi$. Then $m_{t_{j}} \geq m_{i_{1}+j}$ for every $0 \leq j \leq k$. Since $\left\{m_{i_{1}+j}: 0 \leq j \leq k\right\}$ belongs to $S_{\zeta+1}[M]$, which is spreading, we conclude that $\Psi$ belongs to $S_{\zeta+1}[M]$. Our hypothesis (for $\zeta+1$ ) yields that $\psi^{-1}(\Psi)$ is contained in the union of $D$ $S_{\zeta+1}[L]$-sets, hence so is its subset $\left\{l_{i_{1}+j}: 0 \leq j \leq j_{0}, \psi\left(l_{i_{1}+j}\right) \geq m_{i_{1}}\right\}$. On the other hand, the cardinality of $\left\{\psi\left(l_{i_{1}+j}\right): 0 \leq j \leq j_{0}, \psi\left(l_{i_{1}+j}\right)<m_{i_{1}}\right\}$ is at most $i_{1}-1$. Our hypothesis (for $\zeta=0$ ) now yields that the cardinality of the set $\left\{l_{i_{1}+j}: \psi\left(l_{i_{1}+j}\right)<m_{i_{1}}, 0 \leq j \leq j_{0}\right\}$ is at most $D\left(i_{1}-1\right)$. We deduce, since $l_{i_{1}}>i_{1}-1$, that $\left\{l_{i_{1}+j}: \psi\left(l_{i_{1}+j}\right)<m_{i_{1}}, 0 \leq j \leq j_{0}\right\}$ is contained in the union of $D S_{1}[L]$ sets. Hence, $\left\{l_{i_{1}+j}: 0 \leq j \leq j_{0}\right\}$ is contained in the union of $2 D S_{\zeta+1}[L]$ sets.

Next set $j_{1}=\min \left\{j: 0 \leq j \leq q\right.$ and $\left.l_{i_{1}+j}>m_{i_{1}+j}\right\}$. If $j_{1}$ does not exist, then $l_{i_{1}+j} \leq m_{i_{1}+j}$ for all $0 \leq j \leq q$. We deduce, by our previous observation for $j_{0}=q$, that $\left\{l_{i_{1}+j}: 0 \leq j \leq q\right\}$ is contained in the union of $2 D S_{\zeta+1}[L]$ sets.

If $j_{1}$ does exist, then $\left\{l_{i_{1}+j}: 0 \leq j<j_{1}\right\}$ is contained in the union of $2 D S_{\zeta+1}[L]$ sets. Indeed, this is obvious if $j_{1}=0$. If $j_{1} \geq 1$ the assertion follows from our previous observation by taking $j_{0}=j_{1}-1$. Finally; $\left\{l_{i_{1}+j}\right.$ : $\left.j_{1} \leq j \leq q\right\}$ belongs to $S_{\zeta+1}[L]$, since $l_{i_{1}+j_{1}}>m_{i_{1}+j_{1}} \geq m_{i_{1}}$ and $\left\{l_{i_{1}+j}\right.$ : $\left.j_{1} \leq j \leq q\right\}$ is contained in the union of $m_{i_{1}}$ successive maximal $S_{\zeta}[L]$ sets. Thus, $\left\{l_{i_{1}+j}: 0 \leq j \leq q\right\}$ is contained in the union of $2 D+1 S_{\zeta+1}[L]$ sets.

Concluding, in any case the set $\left\{l_{i_{1}+j}: 0 \leq j \leq q\right\}$ is contained in the union of $2 D+1 S_{\zeta+1}[L]$ sets. Hence, applying part 5 of Lemma 3.2, we find that $\tau_{\zeta+1}\left(\left\{l_{i_{1}+j}: 0 \leq j \leq q\right\}\right) \leq(2 D+1)(\zeta+2)+1$, as desired.

Proposition 3.13. Let $\xi<\omega_{1}$ and $L=\left(l_{i}\right), M=\left(m_{i}\right)$ be in $[\mathbb{N}]$. Suppose that there exist $\delta>0$ and a bounded linear operator $T: X_{L}^{\xi} \rightarrow X_{M}^{\xi}$ such that $\left\|T\left(e_{l}^{\xi}\right)\right\|_{0}>\delta$ for every $l \in L$. Then there exist a map $\psi: L \rightarrow M$ and a bounded linear operator $R: X_{L}^{\xi} \rightarrow X_{M}^{\xi}$ such that $R\left(e_{l}^{\xi}\right)=e_{\psi(l)}^{\xi}$ for every $l \in L$.

Proof. Following [11], given two infinite matrices $\left(a_{i j}\right)$ and $\left(d_{i j}\right)$, we shall call $\left(d_{i j}\right)$ a block diagonal of $\left(a_{i j}\right)$ if there exist increasing sequences 
$\left(r_{k}\right),\left(s_{k}\right)$ of positive integers so that

$$
d_{i j}= \begin{cases}a_{i j} & \text { if }(i, j) \in \bigcup_{k=1}^{\infty}\left[r_{k}, r_{k+1}\right) \times\left[s_{k}, s_{k+1}\right), \\ 0 & \text { otherwise. }\end{cases}
$$

We represent $T$ as an infinite matrix $\left(a_{i j}\right)$. Then $T\left(e_{l_{i}}^{\xi}\right)=\sum_{j=1}^{\infty} a_{i j} e_{m_{j}}^{\xi}$ for every $i \in \mathbb{N}$. Because $\left\|T\left(e_{l_{i}}^{\xi}\right)\right\|_{0}>\delta$, for every $i \in \mathbb{N}$ there exists $j \in \mathbb{N}$ such that $\left|a_{i j}\right|>\delta$. We can thus define a map $\psi: L \rightarrow M$ so that if $\psi\left(l_{i}\right)=m_{j}$, then $\left|a_{i j}\right|>\delta$. Observe that $\psi^{-1}\left\{m_{j}\right\}$ is finite for all $j \in \mathbb{N}$, since $\left(T\left(e_{l_{i}}^{\xi}\right)\right)$ is weakly null in $X_{M}^{\xi}$. In particular, $\psi(L) \in[M]$. Let $\left(m_{k_{j}}\right)_{j=1}^{\infty}$ be the increasing enumeration of $\psi(L)$. Given $x=\sum_{i=1}^{\infty} \lambda_{i} e_{l_{i}} \in X_{L}^{\xi}$, we set $S(x)=\sum_{j=1}^{\infty}\left(\sum_{i=1}^{\infty} \lambda_{i} a_{i k_{j}}\right) e_{m_{k_{j}}}^{\xi}$. It follows, since $T$ is bounded and $\left(e_{n}^{\xi}\right)$ is unconditional, that $S$ is a well defined bounded linear operator from $X_{L}^{\xi}$ into $X_{\psi(L)}^{\xi}$. Moreover, the matrix representation $\left(c_{i j}\right)$ of $S$ with respect to the bases $\left(e_{l_{i}}^{\xi}\right)$ and $\left(e_{m_{k_{j}}}^{\xi}\right)$ is given by $c_{i j}=a_{i k_{j}}$ for all positive integers $i, j$.

We next consider the matrix $\left(b_{i j}\right)$ given by

$$
b_{i j}= \begin{cases}c_{i j} & \text { if } \psi\left(l_{i}\right)=m_{k_{j}}, \\ 0 & \text { otherwise. }\end{cases}
$$

Note that there exists a unique non-zero entry in every row of the matrix $\left(b_{i j}\right)$, while each column contains only finitely many non-zero entries. We can thus find a permutation $p$ of $\mathbb{N}$ so that the matrix $\left(b_{p(i) j}\right)$ is a block diagonal of $\left(c_{p(i) j}\right)$. Since $\left(c_{p(i) j}\right)$ represents the bounded linear operator $S: X_{L}^{\xi} \rightarrow X_{\psi(L)}^{\xi}$ with respect to the bases $\left(e_{l_{p(i)}}^{\xi}\right)$ and $\left(e_{m_{k_{j}}}^{\xi}\right)$, and $\left(b_{p(i) j}\right)$ is a block diagonal of $\left(c_{p(i) j}\right)$, Proposition 1.c. 8 of [11] yields that $\left(b_{p(i) j}\right)$ also represents a bounded linear operator from $X_{L}^{\xi}$ into $X_{\psi(L)}^{\xi}$ with respect to the bases $\left(e_{l_{p(i)}}^{\xi}\right)$ and $\left(e_{m_{k_{j}}}^{\xi}\right)$. Consequently, $\left(b_{i j}\right)$ represents a bounded linear operator $W: X_{L}^{\xi} \rightarrow X_{\psi(L)}^{\xi}$ with respect to the bases $\left(e_{l_{i}}^{\xi}\right)$ and $\left(e_{m_{k_{j}}}^{\xi}\right)$ which evidently satisfies $W\left(e_{l_{i}}^{\xi}\right)=a_{i k_{j}} e_{\psi\left(l_{i}\right)}^{\xi}$ (where $\psi\left(l_{i}\right)=m_{k_{j}}$ ) for all $i \in \mathbb{N}$. Because $\left|a_{i k_{j}}\right|>\delta$, if $\psi\left(l_{i}\right)=m_{k_{j}}$, and $\left(e_{n}^{\xi}\right)$ is unconditional, we conclude that there exists a bounded linear operator $R: X_{L}^{\xi} \rightarrow X_{M}^{\xi}$ such that $R\left(e_{l}^{\xi}\right)=e_{\psi(l)}^{\xi}$ for every $l \in L$.

We are now ready for the

Proof of Theorem 1.1. $3 \Rightarrow 1$ and $2 \Rightarrow 3$ are immediate. To prove that 1 implies 2 we first apply Proposition 3.13 to obtain a map $\psi: L \rightarrow M$ and a bounded linear operator $R: X_{L}^{\xi} \rightarrow X_{M}^{\xi}$ such that $R\left(e_{l}^{\xi}\right)=e_{\psi(l)}^{\xi}$ for every $l \in L$. Propositions 3.11 and 3.12 will then yield a constant $E>0$ such that $\tau_{\zeta}\left(\phi^{-1} F\right) \leq E$ for every $F \in S_{\zeta}[M]$ and $0 \leq \zeta \leq \xi$ (where $\phi: L \rightarrow M$ is the natural bijection). The result now follows from Lemma 3.4.
To obtain Corollary 1.2 we shall need the following

LEMMA 3.14. Let $\xi<\omega$ and $s=\left(u_{n}\right)$ be a bounded block basis of $\left(e_{n}^{\xi}\right)$ such that $\lim _{n}\left\|u_{n}\right\|_{0}=0$. Then for every $N \in[\mathbb{N}]$ and $0 \leq \zeta \leq \xi$ there exists $M \in[N]$ so that $\lim _{n}\left\|\zeta_{n}^{M} \cdot s\right\|_{\zeta}=0$. (Given $\mu=\sum_{i=1}^{\infty} a_{i} e_{i} \in c_{00}$, we denote by $\mu \cdot s$ the vector $\sum_{i=1}^{\infty} a_{i} u_{i}$, which of course belongs to $c_{00}$.)

Proof. If $\zeta=0$, the assertion follows from the fact that $\lim _{n}\left\|u_{n}\right\|_{0}=0$. Assume now that $\zeta \leq \xi-1$ and that the assertion holds for $\zeta$. Let $N \in[\mathbb{N}]$ and $\varepsilon>0$. We will find $Q \in[N]$ so that $\left\|(\zeta+1)_{1}^{Q} \cdot s\right\|_{\zeta+1}$ $<\varepsilon$. Once this is accomplished, we can choose $\left(Q_{i}\right) \subset[N]$ so that $\left\|(\zeta+1)_{1}^{Q_{i}} \cdot s\right\|_{\zeta+1}<\varepsilon_{i}$, where $\lim _{i} \varepsilon_{i}=0$ and $F_{1}^{\zeta+1}\left(Q_{1}\right)<F_{1}^{\zeta+1}\left(Q_{2}\right)<\ldots$ Letting $M=\bigcup_{i=1}^{\infty} F_{1}^{\zeta+1}\left(Q_{i}\right)$, we obtain $(\zeta+1)_{1}^{Q_{i}}=(\zeta+1)_{i}^{M}$ for all $i \in \mathbb{N}$, and thus $\lim _{i}\left\|(\zeta+1)_{i}^{M} \cdot s\right\|_{\zeta+1}=0$.

We now pass to the construction of $Q$. By the induction hypothesis we can choose a sequence $\left(P_{i}\right) \subset[N]$ sharing the following properties:

1. $F_{1}^{\zeta}\left(P_{1}\right)<F_{1}^{\zeta}\left(P_{2}\right)<\ldots$

2. $\min F_{1}^{\zeta}\left(P_{1}\right)>(2+2 b) / \varepsilon$, where $b$ is chosen so that $\left\|u_{n}\right\|_{\xi} \leq b$ for every $n \in \mathbb{N}$.

3. $\left\|\zeta_{1}^{P_{i}} \cdot s\right\|_{\zeta}<1 /\left(2^{i} k_{i-1}\right)$ for all $i \geq 2$, where $k_{i}=\max \operatorname{supp}\left(\zeta_{1}^{P_{i}} \cdot s\right)$.

Put $Q=\bigcup_{i=1}^{\infty} F_{1}^{\zeta}\left(P_{i}\right)$. We are going to show that $\left\|\sum_{i=1}^{n} \zeta_{i}^{Q} \cdot s\right\|_{\zeta+1} \leq$ $2+2 b$ for every $n \in \mathbb{N}$. Note that $\zeta_{i}^{Q}=\zeta_{1}^{P_{i}}$ and $F_{i}^{\zeta}(Q)=F_{1}^{\zeta}\left(P_{i}\right)$ for all $i \in \mathbb{N}$.

Let $G \in S_{\zeta+1}$. Let $\left\{i_{1}, \ldots, i_{p}\right\}$ be an enumeration of the set $\{i \leq n$ : $\left.\operatorname{supp}\left(\zeta_{i}^{Q} \cdot s\right) \cap G \neq \emptyset\right\}$. Choose $l \leq \min G$ and $G_{1}<\ldots<G_{l}$ in $S_{\zeta}$ so that $G=\bigcup_{i=1}^{l} G_{i}$. Then $\left|\left(\zeta_{i_{\mathrm{i}}}^{Q} \cdot s\right)(G)\right| \leq b$. Further,

$$
\begin{aligned}
\left|\sum_{t=2}^{p} \zeta_{i_{t}}^{Q} \cdot s(G)\right| & =\left|\sum_{j=1}^{l} \sum_{t=2}^{p}\left(\zeta_{i_{t}}^{Q} \cdot s\right)\left(G_{j}\right)\right| \leq \sum_{j=1}^{l} \sum_{t=2}^{p}\left|\left(\zeta_{i_{t}}^{Q} \cdot s\right)\left(G_{j}\right)\right| \\
& \leq \sum_{j=1}^{l} \sum_{t=2}^{p}\left\|\zeta_{i_{t}}^{Q} \cdot s\right\|_{\zeta} \leq \sum_{j=1}^{l} \sum_{t=2}^{p} \frac{1}{2^{i_{t}} k_{i_{t}-1}} \\
& \leq \frac{l}{k_{i_{1}}} \leq 1, \quad \text { since } l \leq \min G \leq k_{i_{1}} .
\end{aligned}
$$

Therefore, $\left|\sum_{i=1}^{n}\left(\zeta_{i}^{Q} \cdot s\right)(G)\right|=\left|\sum_{t=1}^{p}\left(\zeta_{i_{t}}^{Q} \cdot s\right)(G)\right| \leq b+1$. It follows that $\left\|\sum_{i=1}^{n} \zeta_{i}^{Q} \cdot s\right\|_{\zeta+1} \leq 2+2 b$. If we take $n=\min F_{1}^{\zeta}\left(P_{1}\right)$, we conclude that $\left\|(\zeta+1)_{1}^{Q} \cdot s\right\|_{\zeta+1}<\varepsilon$

Proof of Corollary 1.2. Let $T: X_{L}^{\xi} \rightarrow X_{M}^{\xi}$ be an isomorphic embedding. We apply Theorem 1.1 to show that $\left(e_{l_{n}}^{\xi}\right)$ dominates $\left(e_{m_{n}}^{\xi}\right)$. Indeed, we 
need only check that $\inf _{l \in L}\left\|T\left(e_{l}^{\xi}\right)\right\|_{0}>0$. If that were not the case, let $\left(x_{i}\right)$ be a subsequence of $\left(T\left(e_{l_{i}}^{\xi}\right)\right)$ such that $\lim _{i}\left\|x_{i}\right\|_{0}=0$. By a standard perturbation result we can assume, without loss of generality, that for some block basis $\left(u_{i}\right)$ of $\left(e_{l_{i}}^{\xi}\right)$ and a null sequence of positive scalars $\left(\varepsilon_{i}\right)$ we have $\left\|x_{i}-u_{i}\right\|_{\xi}<\varepsilon_{i}$ for all $i \in \mathbb{N}$. It follows that also $\lim _{i}\left\|u_{i}\right\|_{0}=0$, and thus Lemma 3.14 yields $N \in[\mathbb{N}]$ so that $\lim _{i}\left\|\xi_{i}^{N} \cdot s\right\|_{\xi}=0$, where $s=\left(u_{i}\right)$. But then $\lim _{i}\left\|\xi_{i}^{N} \cdot x\right\|_{\xi}=0$ as well $\left(x=\left(x_{i}\right)\right)$. This is a contradiction because $\left(x_{i}\right)$ is equivalent to a subsequence of $\left(e_{l_{i}}^{\xi}\right)$ and thus it is an $\ell_{1}^{\xi}$-spreading model. Hence, $\left(e_{l_{n}}^{\xi}\right)$ dominates $\left(e_{m_{n}}^{\xi}\right)$, completing the proof of part 1. Parts 2 and 3 are immediate consequences of Theorem 1.1.

We recall that a Banach space $X$ is said to be primary if, for every bounded linear projection $P$ on $X$, either $P X$ or $(I-P) X$ is isomorphic to $X$.

CoRollaRY 3.15. $X_{N}^{\xi}$ is not primary, for every $N \in[\mathbb{N}]$ and all $1 \leq$ $\xi<\omega$.

Proof. We first let $\mathcal{F}=\{(L, M) \in[N] \times[N]: L \cup M=N, L \cap M=\emptyset\}$. Then $\mathcal{F}$ is easily seen to be closed in $[N] \times[N]$ and thus it is a Polish space. We next set $\mathcal{G}=\left\{(L, M) \in \mathcal{F}: d_{\xi}(N, L)=d_{\xi}(N, M)=\infty\right\}$. Arguing as we did in the proof of Lemma 3.5 we deduce that $\mathcal{G}$ is a $G_{\delta}$ dense subset of $\mathcal{F}$. If $(L, M) \in \mathcal{G}$ then $X_{N}^{\xi}=X_{L}^{\xi} \oplus X_{M}^{\xi}$. However, Theorem 1.1 implies that $X_{N}^{\xi}$ is not isomorphic to a subspace of either $X_{L}^{\xi}$ or $X_{M}^{\xi}$.

4. Subspaces spanned by block bases. In this section we investigate subspaces of $X^{\xi}$ spanned by block bases of $\left(e_{n}^{\xi}\right)$. We first show that there exists a block basis of $\left(e_{n}^{\xi}\right)$ spanning a complemented subspace of $X^{\xi}$ which is not isomorphic to $X_{M}^{\zeta}$, for every $M \in[\mathbb{N}]$ and all $0 \leq \zeta \leq \xi$.

LEMMA 4.1. Let $x_{1}<\ldots<x_{p}$ be a finite block basis of $\left(e_{n}\right)$, the unit vector basis of $c_{00}$. Let $G_{1}<\ldots<G_{q}$ be finite subsets of $\mathbb{N}$ and $\left(a_{i}\right)_{i=1}^{p}$ be scalars. Assume that there exists $C>0$ such that $\left|\left(\sum_{i \in I} a_{i} x_{i}\right)\left(\bigcup_{j \in J} G_{j}\right)\right|$ $\leq C$ whenever $I \subset\{1, \ldots, p\}$ and $J \subset\{1, \ldots, q\}$ satisfy one of the following two conditions:

1. $I=\bigcup_{j \in J} I_{j}, I_{j_{1}}<I_{j_{2}}$ if $j_{1}<j_{2}$ and $I_{j}=\left\{i \in I: \operatorname{supp} x_{i} \cap G_{j} \neq \emptyset\right\}$ for all $j \in J$.

2. $J=\bigcup_{i \in I} J_{i}, J_{i_{1}}<J_{i_{2}}$ if $i_{1}<i_{2}$ and $J_{i}=\left\{j \in J: \operatorname{supp} x_{i} \cap G_{j} \neq \emptyset\right\}$ for all $i \in I$.

Then $\left|\left(\sum_{i=1}^{p} a_{i} x_{i}\right)\left(\bigcup_{j=1}^{q} G_{j}\right)\right| \leq 3 C$.

Proof. Given $j \leq q$, we let $T_{j}=\left\{i \leq p: \operatorname{supp} x_{i} \cap G_{j} \neq \emptyset\right\}$. We also let $J=\left\{j \leq q: T_{j} \neq \emptyset\right\}$ and $J_{1}=\left\{j \in J:\left|T_{j}\right|=1\right\}$. Set $J_{2}=J \backslash J_{1}$. Given $j \in J_{2}$ we let $s_{j}=\min T_{j}$ and $t_{j}=\max T_{j}$. We observe that $s_{j_{1}}<t_{j_{1}} \leq s_{j_{2}}$ for every $j_{1}<j_{2}$ in $J_{2}$.

Next, we define a map $\sigma: J_{1} \rightarrow\{1, \ldots, p\}$ so that $\{\sigma(j)\}=T_{j}$ for every $j \in J_{1}$. Note that $\sigma\left(J_{1}\right)$ and $J_{1}$ satisfy condition 2 and therefore

$$
\left|\left(\sum_{i=1}^{p} a_{i} x_{i}\right)\left(\bigcup_{j \in J_{1}} G_{j}\right)\right|=\left|\left(\sum_{i \in \sigma\left(J_{1}\right)} a_{i} x_{i}\right)\left(\bigcup_{j \in J_{1}} G_{j}\right)\right| \leq C .
$$

Suppose now that $J_{2}=\left\{j_{1}, \ldots, j_{k}\right\}$ and put $J_{3}=\left\{j_{r}: r \leq k, r\right.$ is odd $\}$ and $J_{4}=\left\{j_{r}: r \leq k, r\right.$ is even $\}$. It follows that $\bigcup_{j \in J_{m}} T_{j}$ and $J_{m}, m \in\{3,4\}$, satisfy condition 1 and thus

$$
\left|\left(\sum_{i=1}^{p} a_{i} x_{i}\right)\left(\bigcup_{j \in J_{m}} G_{j}\right)\right|=\left|\left(\sum_{i \in \bigcup_{j \in J_{m}} T_{j}} a_{i} x_{i}\right)\left(\bigcup_{j \in J_{m}} G_{j}\right)\right| \leq C, \quad m \in\{3,4\} .
$$

Hence, $\left|\left(\sum_{i=1}^{p} a_{i} x_{i}\right)\left(\bigcup_{j \in J_{2}} G_{j}\right)\right| \leq 2 C$. The assertion follows since

$$
\left(\sum_{i=1}^{p} a_{i} x_{i}\right)\left(\bigcup_{j=1}^{q} G_{j}\right)=\left(\sum_{i=1}^{p} a_{i} x_{i}\right)\left(\bigcup_{j \in J_{1}} G_{j}\right)+\left(\sum_{i=1}^{p} a_{i} x_{i}\right)\left(\bigcup_{j \in J_{2}} G_{j}\right) \text {. }
$$

LEMMA 4.2. Let $1 \leq \zeta \leq \xi<\omega$ and $\left(x_{n}\right)$ be a block basis of $\left(e_{n}^{\xi}\right)$ so that for some $b>0,\left\|x_{n}\right\|_{\xi}<b$, for every $n \in \mathbb{N}$. Let $k_{n}=\max \operatorname{supp} x_{n}$ for every $n \in \mathbb{N}$, and suppose that $\left\|x_{n}\right\|_{\zeta-1}<1 / 2^{k_{n-1}}$ for every $n \geq 2$. Then $\left|\left(\sum_{i=1}^{n} a_{i} x_{i}\right)(H)\right| \leq(2+b) \max _{i \leq n}\left|a_{i}\right|$ for every $H \in S_{\zeta}, n \in \mathbb{N}$ and all scalar sequences $\left(a_{i}\right)_{i=1}^{n}$.

Proof. Let $H \in S_{\zeta}$ and put $i_{0}=\min \left\{i \leq n: \operatorname{supp} x_{i} \cap H \neq \emptyset\right\}$. We may write $H=\bigcup_{j=1}^{r} H_{j}$, where $r \leq \min H$ and $H_{1}<\ldots<H_{r}$ belong to $S_{\zeta-1}$. Note that $\min H I \leq k_{i_{0}}$. We also observe that $\left|x_{i}(H)\right| \leq r\left\|x_{i}\right\|_{\zeta-1}$ and hence

$$
\begin{aligned}
\left|\sum_{i=i_{0}+1}^{n} a_{i} x_{i}(H)\right| & \leq \sum_{i=i_{0}+1}^{n}\left|a_{i}\right| r|| x_{i} \|_{\zeta-1} \\
& \leq r\left(\max _{i \leq n}\left|a_{i}\right|\right) \sum_{i=i_{0}+1}^{\infty} \frac{1}{2^{k_{i-1}}} \leq 2 \max _{i \leq n}\left|a_{i}\right| .
\end{aligned}
$$

Finally, $\left|x_{i_{0}}(H)\right| \leq\left\|x_{i_{0}}\right\|_{\xi}<b$ and so $\left|\left(\sum_{i=1}^{n} a_{i} x_{i}\right)(H)\right| \leq(2+b) \max _{i \leq n}\left|a_{i}\right|$, as desired.

Our next proposition is a partial generalization of Lemma 3.10.

Propositron 4.3. Let $\xi<\omega$ and $\left(x_{n}\right)$ be a semi-normalized block basis of $\left(e_{n}^{\xi}\right)$. Set $\zeta=\min \left\{\alpha \leq \xi: \inf _{n}\left\|x_{n}\right\|_{\alpha}>0\right\}$. Then there exists a subsequence of $\left(x_{n}\right)$ which is equivalent to a subsequence of $\left(e_{n}^{\xi-\zeta}\right)$.

Proof. Choose $\delta>0, b>0$ so that $\delta<\left\|x_{n}\right\|_{\zeta}$ and $\left\|x_{n}\right\|_{\xi}<b$ for every $n \in \mathbb{N}$. Assume first that $\zeta \geq 1$. Then we choose inductively $n_{1}<n_{2}<\ldots$ 
so that $\left\|x_{n_{i}}\right\|_{\zeta-1}<1 / 2^{k_{i-1}}$ for every $i \geq 2$, where $k_{i}=\max \operatorname{supp} x_{n_{i}}$. For every $i \in \mathbb{N}$ we can find $F_{i} \in S_{\zeta}, F_{i} \subset \operatorname{supp} x_{n_{i}}$, so that $\left|x_{n_{i}}\right|\left(F_{i}\right)>\delta$. Put $m_{i}=\min F_{i}$. We are going to show that $\left(x_{n_{i}}\right)$ is equivalent to $\left(e_{m_{i}}^{\xi-\zeta}\right)$. To this end let $k \in \mathbb{N}$ and $\left(a_{i}\right)_{i=1}^{k}$ be scalars. We first show that $\left\|\sum_{i=1}^{k} a_{i} e_{m_{i}}^{\xi-\zeta}\right\| \leq$ $\delta^{-1}\left\|\sum_{i=1}^{k} a_{i} x_{n_{i}}\right\|_{\xi}$. Indeed, if $G \subset\left\{m_{1}, \ldots, m_{k}\right\}$ belongs to $S_{\xi-\zeta}$ then set $A=\left\{i \leq k: m_{i} \in G\right\}$. We have the estimate

$$
\sum_{i \in A}\left|a_{i}\right| \leq \delta^{-1} \sum_{i \in A}\left|a_{i}\right|\left|x_{n_{i}}\right|\left(F_{i}\right) \leq \delta^{-1}\left\|\sum_{i=1}^{k} a_{i} x_{n_{i}}\right\|_{\xi}
$$

as $\bigcup_{i \in A} F_{i} \in S_{\xi}$, by Lemma 3.8 .

Next, let $G \in S_{\xi}$. Lemma 3.8 yields $G_{1}<\ldots<G_{q}$ in $S_{\zeta}$ with $\left\{\min G_{j}\right.$ : $j \leq q\}$ belonging to $S_{\xi-\zeta}$ and such that $G=\bigcup_{j=1}^{q} G_{j}$. We shall apply Lemma 4.1 in order to estimate $\left|\left(\sum_{i=1}^{k} a_{i} x_{n_{i}}\right)\left(\bigcup_{j=1}^{q} G_{j}\right)\right|$. Let $I \subset\{1, \ldots, k\}$ and $J \subset\{1, \ldots, q\}$ satisfy condition 1 of Lemma 4.1. Then $I_{j}=\{i \in$ $\left.I: \operatorname{supp} x_{n_{i}} \cap G_{j} \neq \emptyset\right\}$ for every $j \in J$. We choose $i_{j} \in I_{j}$ such that $\left|a_{i_{j}}\right|=\max _{i \in I_{j}}\left|a_{i}\right|$ for every $j \in J$. Fix $j_{0} \in J$. Then

$$
\begin{aligned}
\left|\left(\sum_{i \in I_{j_{0}}} a_{i} x_{n_{i}}\right)\left(\bigcup_{j \in J} G_{j}\right)\right| & =\left|\sum_{i \in I_{j_{0}}} a_{i} x_{n_{i}}\left(G_{j_{0}}\right)\right| \leq(2+b) \max _{i \in I_{j_{0}}}\left|a_{i}\right| \\
& =(2+b)\left|a_{i_{j_{0}}}\right|, \quad \text { by Lemma } 4.2 .
\end{aligned}
$$

Hence $\left|\left(\sum_{i \in I} a_{i} x_{n_{i}}\right)\left(\bigcup_{j \in J} G_{j}\right)\right| \leq(2+b) \sum_{j \in J}\left|a_{i_{j}}\right|$.

Note also that $\left\{m_{i_{j}}: j \in J \backslash\{\min J\}\right\}$ belongs to $S_{\xi-\zeta}$. This is so since supp $x_{n_{i}} \cap G_{j} \neq \emptyset$ whenever $i \in I_{j}$ and $j \in J$, and thus $\min G_{j_{1}}<$ $\min \operatorname{supp} x_{n_{i}} \leq m_{i}$ for every $i \in I_{j_{2}}$ and $j_{1}<j_{2}$ in $J$. In particular, $\min G_{j_{1}}<m_{i_{j}}$ when $j_{1}<j_{2}$ in $J$. Since $S_{\xi-\zeta}$ is spreading we see that $\left\{m_{i_{j}}\right.$ : $j \in J \backslash\{\min J\}\}$ belongs to $S_{\xi-\zeta}$. It now follows that $\sum_{j \in J \backslash\{\min J\}}\left|a_{i_{j}}\right| \leq$ $\left\|\sum_{i=1}^{k} a_{i} e_{m_{i}}^{\xi-\zeta}\right\|$ and hence

$$
\left|\left(\sum_{i \in I} a_{i} x_{n_{i}}\right)\left(\bigcup_{j \in J} G_{j}\right)\right| \leq 2(2+b)\left\|\sum_{i=1}^{k} a_{i} e_{m_{i}}^{\xi-\zeta}\right\| .
$$

We now assume that $I \subset\{1, \ldots, k\}$ and $J \subset\{1, \ldots, q\}$ satisfy condition 2 of Lemma 4.1. Then $J_{i}=\left\{j \in J: \operatorname{supp} x_{n_{i}} \cap G_{j} \neq \emptyset\right\}$ for all $i \in I$. An argument similar to that in the preceding paragraph yields that $\left\{m_{i}: i \in\right.$ $I \backslash\{\min I\}\}$ belongs to $S_{\xi-\zeta}$. It follows that $\sum_{i \in I}\left|a_{i}\right| \leq 2\left\|\sum_{i=1}^{k} a_{i} e_{m_{i}}^{\xi-\zeta}\right\|$. Finally,

$$
\begin{aligned}
\left|\left(\sum_{i \in I} a_{i} x_{n_{i}}\right)\left(\bigcup_{j \in J} G_{j}\right)\right| & =\left|\sum_{i \in I} a_{i} x_{n_{i}}\left(\bigcup_{j \in J_{i}} G_{j}\right)\right| \leq b \sum_{i \in I}\left|a_{i}\right| \text { as } \bigcup_{j \in J_{i}} G_{j} \in S_{\xi} \\
& \leq 2 b\left\|\sum_{i=1}^{k} a_{i} e_{m_{i}}^{\xi-\zeta}\right\| .
\end{aligned}
$$

We deduce from Lemma 4.1 that

$$
\left|\left(\sum_{i=1}^{k} a_{i} x_{n_{i}}\right)\left(\bigcup_{j=1}^{q} G_{j}\right)\right| \leq 6(2+b)\left\|\sum_{i=1}^{k} a_{i} e_{m_{i}}^{\xi-\zeta}\right\|,
$$

and hence $\left\|\sum_{i=1}^{k} a_{i} x_{n_{i}}\right\|_{\xi} \leq 12(2+b)\left\|\sum_{i=1}^{k} a_{i} e_{m_{i}}^{\xi-\zeta}\right\|$.

To complete the proof we need to consider the case $\zeta=0$. We now choose $m_{n} \in \operatorname{supp} x_{n}$ such that $\left|x_{n}\right|\left(\left\{m_{n}\right\}\right)>\delta$ for all $n \in \mathbb{N}$. We are going to show that $\left(x_{n}\right)$ is equivalent to $\left(e_{m_{n}}^{\xi}\right)$. Arguing as we did in the case $\zeta \geq 1$ we find that $\left\|\sum_{i=1}^{k} a_{i} e_{m_{i}}^{\xi}\right\| \leq \delta^{-1}\left\|\sum_{i=1}^{k} a_{i} x_{i}\right\|_{\xi}$ for every $k \in \mathbb{N}$ and all scalar sequences $\left(a_{i}\right)_{i=1}^{k}$.

Next let $G \in S_{\xi}$ and put $I=\left\{i \leq k: \operatorname{supp} x_{i} \cap G \neq \emptyset\right\}$. Then

$$
\begin{aligned}
\left|\sum_{i=1}^{k} a_{i} x_{i}(G)\right| & \leq \sum_{i \in I}\left|a_{i}\right|\left|x_{i}(G)\right| \leq b \sum_{i \in I}\left|a_{i}\right| \\
& \leq 2 b\left\|\sum_{i=1}^{k} a_{i} e_{m_{i}}^{\xi}\right\| \quad \text { as }\left\{m_{i}: i \in I \backslash\{\min I\}\right\} \in S_{\xi} .
\end{aligned}
$$

Hence $\left\|\sum_{i=1}^{k} a_{i} x_{i}\right\|_{\xi} \leq 4 b\left\|\sum_{i=1}^{k} a_{i} e_{m_{i}}^{\xi}\right\|$.

As an immediate consequence of Proposition 4.3 we obtain

COROLLARY 4.4. For every semi-normalized weakly null sequence in $X^{\xi}$, $\xi<\omega$, there exist $\zeta \leq \xi$ and a subsequence which is equivalent to a subsequence of $\left(e_{n}^{\zeta}\right)$.

LEMMA 4.5. Let $1 \leq \xi<\omega$ and $\left(F_{n}\right)$ be a sequence of successive members of $S_{\xi}$ satisfying the following requirements:

1. $\left(\tau_{\xi-1}\left(F_{n}\right)\right)$ increases to $\infty$.

2. $\sup _{n} \frac{\min F_{n}}{\tau_{\xi-1}\left(F_{n+k}\right)}>k$ for every $k \in \mathbb{N}$.

Let $\left(u_{n}\right)$ be a convex block basis of $\left(e_{n}^{\xi}\right)$ such that supp $u_{n}=F_{n}$ for every $n \in \mathbb{N}$. Assume furthermore that $\sum_{n=1}^{\infty}\left\|u_{n}\right\|_{\xi-1}<\infty$. Then the closed linear span of $\left(u_{n}\right)$ in $X^{\xi}$ is not isomorphic to $X_{M}^{\zeta}$, for every $\zeta \leq \xi$ and $M \in[\mathbb{N}]$.

Proof. $\left(u_{n}\right)$ is normalized in $X^{\xi}$ since $F_{n} \in S_{\xi}$ for every $n \in \mathbb{N}$. We let $X$ denote the closed linear span of $\left(u_{n}\right)$ in $X^{\xi}$. Because $\sum_{n=1}^{\infty}\left\|u_{n}\right\|_{\xi-1}<\infty$, we deduce from Proposition 4.3 that every semi-normalized block basis of $\left(u_{n}\right)$ admits a subsequence equivalent to the unit vector basis of $c_{0}$. Indeed, let $\left(v_{n}\right), v_{n}=\sum_{i \in G_{n}} b_{i} u_{i}$, be a semi-normalized block basis of $\left(u_{n}\right)$. Note that $\left(b_{n}\right)$ is bounded since $\left(v_{n}\right)$ is. But also, $\lim _{n} \sum_{i \in G_{n}}\left\|u_{i}\right\|_{\xi-1}=0$, since $\sum_{n=1}^{\infty}\left\|u_{n}\right\|_{\xi-1}<\infty$. Therefore $\lim _{n}\left\|v_{n}\right\|_{\xi-1}=0$ and hence Proposition 4.3 (for $\zeta=\xi$ ) yields a subsequence of $\left(v_{n}\right)$ equivalent to the unit basis of $c_{0}$. 
It follows that every semi-normalized weakly null sequence in $X$ admits a subsequence equivalent to the unit vector basis of $c_{0}$. That is, $X$ has property $(S)$ (see [7]). However, $X_{M}^{\zeta}$ fails property $(S)$ when $\zeta \geq 1$ and $M \in[\mathbb{N}]$. Thus, $X_{M}^{\zeta}$ is not isomorphic to a subspace of $X$, for every $1 \leq \zeta \leq \xi$ and $M \in[\mathbb{N}]$.

To complete the proof we show that $X$ is not isomorphic to $c_{0}$. This is accomplished by showing that for every $k \in \mathbb{N}$ there exists $n \in \mathbb{N}$ so that $\left(u_{n+i}\right)_{i=1}^{k}$ is isometrically equivalent to the unit vector basis of $\ell_{1}^{k}$. In particular, $X$ contains uniformly complemented $\ell_{1}^{k}$ 's. It is a well known fact that $c_{0}$ fails this property.

We let $k \in \mathbb{N}$ and choose $n \in \mathbb{N}$ according to 2 so that

$$
\frac{\min F_{n}}{\tau_{\xi-1}\left(F_{n+k}\right)}>k
$$

Condition 1 now yields that $\sum_{i=1}^{k} \tau_{\xi-1}\left(F_{n+i}\right)<\min F_{n}$ and thus $\bigcup_{i=1}^{k} F_{n+i}$ $\in S_{\xi}$. Hence

$$
\left\|\sum_{i=1}^{k} a_{i} u_{n+i}\right\|_{\xi} \geq \sum_{i=1}^{k}\left|a_{i}\right| u_{n+i}\left(F_{n+i}\right)=\sum_{i=1}^{k}\left|a_{i}\right|
$$

for every scalar sequence $\left(a_{i}\right)_{i=1}^{k}$. Therefore $\left(u_{n+i}\right)_{i=1}^{k}$ is isometrically equivalent to the unit vector basis of $\ell_{1}^{k}$.

Proposition 4.6. Let $1 \leq \xi<\omega$. There exists a normalized convex block basis $\left(u_{n}\right)$ of $\left(e_{n}^{\xi}\right)$ so that letting $F_{n}=\operatorname{supp} u_{n}$ for every $n \in \mathbb{N}$, the following hold:

1. $\tau_{\xi-1}\left(F_{n}\right)=n^{2}$ and $\min F_{n}>k(n+k)^{2}$ for every $n$ and $k$ in $\mathbb{N}$ such that $k<n$.

2. $X=\left[u_{n}: n \in \mathbb{N}\right]$ is not isomorphic to $X_{M}^{\zeta}$ for every $\zeta \leq \xi$ and $M \in[\mathbb{N}]$.

3. $X$ is complemented in $X^{\xi}$.

Pr o of. We inductively choose a sequence of successive integer intervals $\left(F_{n}\right)$ such that for every $n \in \mathbb{N}$,

$$
\min F_{n}>\max \left\{k(n+k)^{2}: k<n\right\} \quad \text { and } \tau_{\xi-1}\left(F_{n}\right)=n^{2} .
$$

We now let, for every $n \in \mathbb{N}$,

$$
M_{n}=F_{n} \cup\left\{m \in \mathbb{N}: m>\max F_{n}\right\} \quad \text { and } \quad u_{n}=\frac{1}{n^{2}} \sum_{i=1}^{n^{2}}(\xi-1)_{i}^{M_{n}}
$$

Condition 1 is an immediate consequence of the inductive construction. This condition implies that in fact $F_{n} \in S_{\xi}$ for every $n \in \mathbb{N}$, and thus $\left(u_{n}\right)$ is indeed a normalized convex block basis of $\left(e_{n}^{\xi}\right)$. We also deduce from
Lemma 2.3 that $\left\|u_{n}\right\|_{\xi-1} \leq \xi / n^{2}$ and so $\sum_{n=1}^{\infty}\left\|u_{n}\right\|_{\xi-1}<\infty$. Hence condition 2 holds in view of Lemma 4.5 .

It remains to establish that $X$ is complemented in $X^{\xi}$. To this end we define a map $P: c_{00} \rightarrow c_{00}$ by

$$
P(x)=\sum_{i=1}^{\infty} x\left(F_{i}\right) u_{i} \quad \text { for all } x \in c_{00}
$$

Clearly, $P$ is well defined and linear. It is also clear that $P\left(u_{i}\right)=u_{i}$ for every $i \in \mathbb{N}$. Our objective is to show that $P$ is bounded with respect to the $\|\cdot\|_{\xi}$-norm on $c_{00}$, for then $P$ will extend to a bounded linear projection on $X^{\xi}$ with range equal to $X$. To achieve our goal it suffices to show that if $G \in S_{\xi}$ is maximal, then $\left(\sum_{i=1}^{p} x\left(F_{i}\right) u_{i}\right)(G) \leq 18 \xi$ for every $p \in \mathbb{N}$ and $x \in c_{00},\|x\|_{\xi} \leq 1$, with $x(\{i\}) \geq 0, i \in \mathbb{N}$.

According to condition 1 of our hypothesis, for every $i \in \mathbb{N}$ there exist successive $S_{\xi-1}$ sets $F_{i 1}<\ldots<F_{i i^{2}}$ so that $F_{i}=\bigcup_{k=1}^{i^{2}} F_{i k}$, and $\left\{\min F_{i k}\right.$ : $\left.k \leq i^{2}\right\} \in S_{1}$. Next let $q=\min G$ and choose maximal members $G_{1}<\ldots$ $<G_{q}$ of $S_{\xi-1}$ so that $G=\bigcup_{j=1}^{q} G_{j}$. Of course, $\left\{\min G_{j}: j \leq q\right\}$ is maximal in $S_{1}$. We shall apply Lemma 4.1. Let $I \subset\{1, \ldots p\}$ and $J \subset\{1, \ldots q\}$ satisfy condition 1 of Lemma 4.1. Recall that $I_{j}=\left\{i \in I: \operatorname{supp} u_{i} \cap G_{j} \neq \emptyset\right\}$, $j \in J$. For each $j \in J$ we choose $i_{j} \in I_{j}$ and $k_{j} \leq i_{j}^{2}$ such that $x\left(F_{i_{j} k_{j}}\right)=$ $\max _{k \leq i^{2}, i \in I_{j}} x\left(F_{i k}\right)$. We have the estimate

$$
\begin{aligned}
\left(\sum_{i \in I} x\left(F_{i}\right) u_{i}\right)\left(\bigcup_{j \in J} G_{j}\right) & =\sum_{j \in J} \sum_{i \in I_{j}} x\left(F_{i}\right) u_{i}\left(G_{j}\right) \\
& =\sum_{j \in J} \sum_{i \in I_{j}} x\left(F_{i}\right) \frac{1}{i^{2}} \sum_{k=1}^{i^{2}}(\xi-1)_{k}^{M_{i}}\left(G_{j}\right) \\
& \leq \sum_{j \in J} \sum_{i \in I_{j}} x\left(F_{i_{j} k_{j}}\right) \sum_{k=1}^{i^{2}}(\xi-1)_{k}^{M_{i}}\left(G_{j}\right) \\
& \leq \sum_{j \in J} x\left(F_{i_{j} k_{j}}\right) \sum_{i \in I_{j}} \sum_{k=1}^{i^{2}}(\xi-1)_{k}^{M_{i}}\left(G_{j}\right) \\
& \leq \sum_{j \in J} x\left(F_{i_{j} k_{j}}\right) \xi \text { by Lemma } F_{i}=\bigcup_{k=1}^{i^{2}} F_{i k} \\
& \leq \xi x\left(\bigcup_{j \in J} F_{i_{j} k_{j}}\right) \leq 2 \xi .
\end{aligned}
$$


The last inequality holds because $\|x\|_{\xi} \leq 1$ and $\bigcup_{j \in J \backslash\{\min J\}} F_{i_{j} k_{j}} \in S_{\xi}$. Indeed, $\min G_{j_{1}}<\min F_{i_{j_{2}} k_{j_{2}}}$ when $j_{1}<j_{2}$ in $J$ and therefore, as $\left\{\min G_{j}\right.$ : $j \leq q\} \in S_{1}, \bigcup_{j \in J \backslash\{\min J\}} F_{i_{j} k_{j}}$ belongs to $S_{\xi}$ by Lemma 3.8 .

Next assume that $I \subset\{1, \ldots, p\}$ and $J \subset\{1, \ldots, q\}$ satisfy condition 2 of Lemma 4.1. Then $J_{i}=\left\{j \in J: \operatorname{supp} u_{i} \cap G_{j} \neq \emptyset\right\}, i \in I$. We set $H_{i}=\{j \in$ $\left.J_{i}: G_{j} \subset \operatorname{supp} u_{i}\right\}, i \in I$. Since supp $u_{i}=F_{i}$ is an interval, $\left|J_{i}\right| \leq\left|H_{i}\right|+2$ for all $i \in I$. Moreover, since each $G_{j}$ is a maximal $S_{\xi-1}$ set and $\tau_{\xi-1}\left(F_{i}\right)=i^{2}$, we have $\left|H_{i}\right| \leq i^{2}$ for all $i \in I$. To estimate $\sum_{i \in I} \sum_{j \in J_{i} \backslash H_{i}} x\left(F_{i}\right) u_{i}\left(G_{j}\right)$, choose $j_{i} \in J_{i} \backslash H_{i}$ for every $i \in I$ (we have assumed without loss of generality that $\left.J_{i} \backslash H_{i} \neq \emptyset\right)$. Then the sets $I$ and $\left\{j_{i}: i \in I\right\}$ satisfy condition 1 of Lemma 4.1. We deduce from our preceding work that

$$
\sum_{i \in I} \sum_{j \in J_{i} \backslash H_{i}} x\left(F_{i}\right) u_{i}\left(G_{j}\right) \leq 4 \xi
$$

as $\left|J_{i} \backslash H_{i}\right| \leq 2$ for every $i \in I$. We next choose, for every $i \in I, R_{i} \subset$ $\left\{1, \ldots, i^{2}\right\}$ with $\left|R_{i}\right|=\left|H_{i}\right|$ and such that

$$
\frac{1}{i^{2}} \sum_{k=1}^{i^{2}} x\left(F_{i k}\right) \leq \frac{1}{\left|H_{i}\right|} \sum_{k \in R_{i}} x\left(F_{i k}\right) .
$$

This choice is possible since $\left|H_{i}\right| \leq i^{2}$. (We make use of the following fact: Let $\left(a_{i}\right)_{i=1}^{n}$ be scalars with $a_{i} \leq a_{j}, i \leq j$, and let $k<n$. Then $(1 / n) \sum_{i=1}^{n} a_{i} \leq$ $(1 /(n-k)) \sum_{i=k+1}^{n} a_{i}$.) We now have

$$
\begin{aligned}
\sum_{i \in I} \sum_{j \in H_{i}} x\left(F_{i}\right) u_{i}\left(G_{j}\right) & =\sum_{i \in I} \sum_{j \in H_{i}}\left[\sum_{k=1}^{i^{2}} x\left(F_{i k}\right)\right]\left[\frac{1}{i^{2}} \sum_{k=1}^{i^{2}}(\xi-1)_{k}^{M_{i}}\left(G_{j}\right)\right] \\
& \leq \sum_{i \in I} \sum_{j \in H_{i}}\left[\frac{1}{i^{2}} \sum_{k=1}^{i^{2}} x\left(F_{i k}\right)\right] \xi \quad \text { by Lemma } 2.3 \\
& \leq \xi \sum_{i \in I} \sum_{j \in H_{i}} \frac{1}{\left|H_{i}\right|} \sum_{k \in R_{i}} x\left(F_{i k}\right) \leq \xi \sum_{i \in I} \sum_{k \in R_{i}} x\left(F_{i k}\right) \\
& \leq \xi x\left(\bigcup_{i \in I, k \in R_{i}} F_{i k}\right) \leq 2 \xi .
\end{aligned}
$$

The last inequality follows since $\|x\|_{\xi} \leq 1$ and $\bigcup_{i \in I \backslash\{\min I\}, k \in R_{i}} F_{i k} \in S_{\xi}$. Indeed, the cardinality of the set $\left\{\min F_{i k}: k \in R_{i}, i \in I\right\}$ does not exceed that of $J$ since $\left|R_{i}\right|=\left|H_{i}\right|$ for all $i \in I$. It now follows, since $|J| \leq \min G_{1}$, that $\left\{\min F_{i k}: k \in R_{i}, i \in I \backslash\{\min I\}\right\}$ belongs to $S_{1}$ and thus $\bigcup_{i \in I, k \in R_{i}} F_{i k}$ is the union of two members of $S_{\xi}$. Concluding,

$$
\begin{aligned}
\left(\sum_{i \in I} x\left(F_{i}\right) u_{i}\right)\left(\bigcup_{j \in J} G_{j}\right) & =\sum_{i \in I}\left[\sum_{j \in J_{i} \backslash H_{i}} x\left(F_{i}\right) u_{i}\left(G_{j}\right)+\sum_{j \in H_{i}} x\left(F_{i}\right) u_{i}\left(G_{j}\right)\right] \\
& \leq 4 \xi+2 \xi=6 \xi .
\end{aligned}
$$

Lemma 4.1 now implies that $\left(\sum_{i=1}^{p} x\left(F_{i}\right) u_{i}\right)(G) \leq 18 \xi$ for every $p \in \mathbb{N}$ and $x \in c_{00},\|x\|_{\xi} \leq 1$, with $x(\{i\}) \geq 0, i \in \mathbb{N}$. It follows that $\|P\| \leq 18 \xi$. The proof of the proposition is now complete.

Proposition 4.7. Let $1 \leq \xi<\omega$ and $\left(u_{n}\right)$ be a block basis of $\left(e_{n}^{\xi}\right)$ such that

1. $u_{n}=v_{n}+w_{n}$ with $\operatorname{supp} v_{n} \cap \operatorname{supp} w_{n}=\emptyset, n \in \mathbb{N}$.

2. $\left(w_{n}\right)$ is equivalent to the unit vector basis of $c_{0}$.

3. $\lim _{n}\left\|v_{n}\right\|_{\xi}=0$ yet $\sup _{n}\left\|\sum_{i=1}^{n} v_{i}\right\|_{\xi}=\infty$.

Then there exists no projection from $X^{\xi}$ onto the closed linear span of $\left(u_{n}\right)$.

Proof. Let $X$ denote the closed linear span of $\left(u_{n}\right)$ in $X^{\xi}$ and assume that $P: X^{\xi} \rightarrow X$ is a bounded linear projection. Note that since $\left(e_{n}^{\xi}\right)$ is unconditional our assumptions yield that $\left(u_{n}\right)$ is semi-normalized in $X^{\xi}$. Lemma 2.a.11 of [11] now implies that $\left(w_{n}\right)$ dominates $\left(v_{n}\right)$, contradicting 3 as $\sup _{n}\left\|\sum_{i=1}^{n} w_{i}\right\|_{\xi}<\infty$

It is easy to construct a normalized convex block basis of $\left(e_{n}^{\xi}\right), \xi \geq 1$, satisfying conditions $1-3$. Indeed, let $M \in[\mathbb{N}], M=\left(m_{n}\right)$, such that $\sum_{n}\left(1 / m_{n}\right)<\infty$. Let $q_{n}=\min F_{n}^{\xi}(M), n \in \mathbb{N}$ (recall that $F_{n}^{\xi}(M)=$ supp $\left.\xi_{n}^{M}\right)$. Because $\xi \geq 1,\left(e_{q_{n}}^{\xi}\right)$ is not dominated by the unit vector basis of $c_{0}$. It follows that there exists a sequence $\left(a_{n}\right)$ of positive scalars such that $\lim _{n} a_{n}=0$ and $\sup _{n}\left\|\sum_{i=1}^{n} a_{i} e_{q_{i}}^{\xi}\right\|=\infty$. Set

$$
v_{n}=a_{n} e_{q_{n}}^{\xi} \quad \text { and } \quad w_{n}=\frac{1-a_{n}}{1-\xi_{n}^{M}\left(q_{n}\right)} \sum_{i \in F_{n}^{\xi}(M) \backslash\left\{q_{n}\right\}} \xi_{n}^{M}(i) e_{i}^{\xi}, \quad n \in \mathbb{N} \text {. }
$$

Finally, we let $u_{n}=v_{n}+w_{n}, n \in \mathbb{N}$. Evidently, $\left(u_{n}\right)$ is a normalized convex block basis of $\left(e_{n}^{\xi}\right)$ satisfying 1 and 3 . It remains to show that 2 holds. Since $\sum_{n} 1 / m_{n}<\infty$ and $\left(\xi_{n}^{M}\right)$ is equivalent to the unit vector basis of $c_{0}$, letting $x_{n}=\sum_{i \in F_{n}^{\xi}(M) \backslash\left\{q_{n}\right\}} \xi_{n}^{M}(i) e_{i}^{\xi}$, we have $\sup _{n}\left\|\sum_{i=1}^{n} x_{i}\right\|_{\xi}<\infty$. It follows that $\left(w_{n}\right)$ is equivalent to the unit vector basis of $c_{0}$ as $\lim _{n} a_{n}=0$ and $\lim _{n} \xi_{n}^{M}\left(q_{n}\right)=0$.

\section{References}

[1] D. E. Alspach and S. A. Argyros, Complexity of weakly null sequences, Dissertationes Math. 321 (1992). 


\section{INFORMATION FOR AUTHORS}

G. Androulakis and E. Odell, Distorting mixed Tsirelson spaces, Israel J. Math. 109 (1999), 125-149.

[3] S. A. Argyros and I. Deliyanni, Examples of asymptotic $l_{1}$ Banach spaces, Trans. Amer. Math. Soc. 349 (1997), 973-995.

[4] S. A. Argyros and V. Felouzis, Interpolating hereditarily indecomposable Banach spaces, J. Amer. Math. Soc. 13 (2000), 243-294.

55] S. A. Argyros and I. Gasparis, Unconditional structures of weakly null sequences, Trans. Amer. Math. Soc., to appear.

[6] S. A. Argyros, S. Mercourakis and A. Tsarpalias, Convex unconditionality and summability of weakly null sequences, Israel J. Math. 107 (1998), 157-193.

[7] P. Cembranos, The hereditary Dunford-Pettis property on $C(K, E)$, Illinois J. Math. 31 (1987), 365-373.

[8] I. Gasparis, A dichotomy theorem for subsets of the power set of the natural numbers, Proc. Amer. Math. Soc., to appear.

[9] A. Kechris, Classical Descriptive Set Theory, Grad. Texts in Math. 156, Springer, New York, 1994.

[10] K. Kuratowski, Applications of the Baire-category method to the problem of independent sets, Fund. Math. 81 (1973), 65-72.

[11] J. Lindenstrauss and L. Tzafriri, Classical Banach Spaces I, $I I$, Ergeb. Math. Grenzgeb. 92, 97, Springer, Berlin, 1977, 1979

[12] J. Myciels ki, Almost every function is independent, Fund. Math. 81 (1973), 43-48.

[13] E. Odell, On quotients of Banach spaces having shrinking unconditional bases, Illinois J. Math. 36 (1992), 681-695.

[14] E. Odell, N. Tomczak-Jaegermann and R. Wagner, Proximity to $\ell_{1}$ and distortion in asymptotic $\ell_{1}$ spaces, J. Funct. Anal. 150 (1997), 101-145.

[15] J. Schreier, Ein Gegenbeispiel zur Theorie der schwachen Konvergenz, Studia Math. 2 (1930), 58-62.

Department of Mathematics

Oklahoma State University

Stillwater, OK 74078-1058, U.S.A.

E-mail: ioagaspa@math.okstate.edu

Department of Mathematics National University of Singapore Singapore 117543

E-mail: matlhh@nus.edu.sg Revised version February 14, 2000

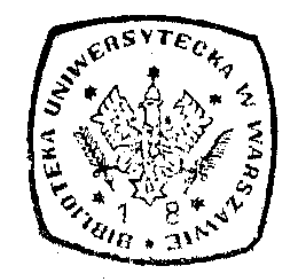

Manuscripts should be typed on one side only, with double or triple spacing and wide margins, and submitted in duplicate, including the original typewritten copy.

An abstract of not more than 200 words and the AMS Mathematics Subject Classification are required.

Figures must be prepared in a form suitable for direct reproduction. Sending EPS, PCX, TIF or CorelDraw files will be most helpful. The author should indicate on the margin of the manuscript where figures are to be inserted.

References should be arranged in alphabetical order, typed with double spacing, and styled and punctuated according to the examples given below. Abbreviations of journal mames should follow Mathematical Reviews. Titles of papers in Russian should be translated into English.

Examples:

[6] D. Beck, Introduction to Dynamical Systems, Vol. 2, Progr. Math. 54, Birkhäuser, Basel, 1978.

[7] R. Hill and A. James, An index formula, J. Differential Equations 15 (1982), 197-211.

[8] J. Kowalski, Some remarks on $J(X)$, in: Algebra and Analysis (Edmonton, 1973), E. Brook (ed.), Lecture Notes in Math. 867, Springer, Berlin, 1974, 115-124.

[Nov] A. S. Novikov, An existence theorem for planar graphs, preprint, Moscow University, 1980 (in Russian).

Authors' affiliation should be given at the end of the manuscript.

Authors receive only page proofs (one copy). If the proofs are not returned promptly, the article will be printed in a later issue.

Authors receive 50 reprints of their articles. Additional reprints can be ordered.

The publisher strongly encourages submission of manuscripts written in TEX. On acceptance of the paper, authors will be asked to transmit the file by electronic mail to: STUDIA@IMPAN.GOV.PL

Recommended format of manuscripts: 12-point type (including references), text width $13.5 \mathrm{~cm}$.

Home page: http://www.impan.gov.pl/PUBL/ 